\title{
Step-tracking Movements of the Wrist. III. Influence of Changes in Load on Patterns of Muscle Activity
}

\author{
Donna S. Hoffman and Peter L. Strick \\ Research Service, V.A. Medical Center and Departments of Neurosurgery and Physiology, SUNY Health Science Center \\ at Syracuse, Syracuse, New York 13210
}

Human subjects performed step-tracking movements of the wrist in the radial direction. Movement amplitude, external load, and accuracy instructions were varied. We used surface electrodes to record muscle activity from an agonist, extensor carpi radialis longus, and an antagonist, extensor carpi ulnaris.

When subjects performed movements "as fast as possible" that were opposed by different external loads, we observed two distinct patterns of modulation of the agonist burst. In one pattern, termed pulse-height modulation, the force of the agonist muscle was graded by varying the peak amplitude of a short-duration agonist burst. This pattern occurred when subjects performed movements of different amplitudes with a lightweight manipulandum. In the other pattern, termed pulse-width modulation, the force of the agonist muscle was graded by varying the duration of an agonist burst of nearly maximal amplitude. When the agonist burst was prolonged, the onset of antagonist activity was delayed. This pattern occurred when subjects performed movements of different amplitudes that were opposed by elastic or viscoelastic loads applied to a heavy manipulandum. The strongest subject exhibited more pulse-height modulation and less pulse-width modulation of the agonist burst than other subjects. Conversely, the weakest subject displayed more pulse-width modulation of the agonist burst than other subjects. These observations indicate that the force requirements of a task, relative to the force generating capacity of a subject's agonist muscle(s), have a significant influence on the pattern of agonist modulation.

In a second experiment using three nonhuman primates, we observed that agonist bursts in wrist flexor and extensor muscles exhibited strikingly different patterns of modulation. For wrist flexion, agonist bursts in wrist flexors were brief and displayed pulse-height modulation when movement amplitude was varied. For wrist extension, agonist bursts in wrist extensors were prolonged and displayed largely pulsewidth modulation when movement amplitude was varied. We

Received Jan. 4, 1993; revised June 3, 1993; accepted June 10, 1993.

This work was supported by funds from the Velerans Administration Medical Research Service. We thank Dr. Michael R. Stiles for his expert technical assistance with the development of the electronics for torque motor control and participation in the initial experiments.

Correspondence should be addressed to Dr. Peter L. Strick, Research Service (151), V.A. Medical Center, Syracuse, NY 13210.

Copyright $(\mathcal{C} 1993$ Society for Neuroscience $0270-6474 / 93 / 135212-16 \$ 05.00 / 0$ suggest that the distinct patterns of modulation observed in the wrist muscles of monkeys were due to differences in the strength of wrist flexors and extensors, rather than to alterations in movement strategy.

In a third experiment, we instructed human subjects to be "accurate" when they made step-tracking movements. When subjects performed movements with a lightweight manipulandum, most displayed short-duration agonist bursts that were pulse-height modulated. When subjects performed "accurate" movements that were opposed by elastic loads, they displayed pulse-width modulation of a small-amplitude agonist burst. This result indicates that the duration of the agonist burst can be modulated even when the amplitude of the burst is not at its maximum.

These findings, together with those of our prior study (Hoffman and Strick, 1990), demonstrate that the nervous system can independently specify three parameters of agonist and antagonist muscle activity: (1) the amplitude of an agonist burst, (2) the duration of an agonist burst, and (3) the amplitude of an antagonist burst. This flexibility over the control of agonist and antagonist activity enables the nervous system to shape precisely the magnitude and time course of the force needed to accomplish a specific task.

[Key words: wrist movements, step-tracking movements, EMG, muscle activity, agonist, antagonist, motor control, motor systems]

This article represents a continuation of our studies on the control of step-tracking movements of the wrist (Hoffman and Strick, 1986a,b, 1990). It is well known that these movements are associated with alternating phasic bursts in agonist and antagonist muscles. The magnitude and timing of the initial agonist and antagonist bursts have been analyzed in an effort to deduce the underlying rules by which these bursts are governed. On the surface, it appears that the rules for distal movements differ from those for proximal movements. In general, when subjects perform finger or wrist movements of different amplitudes, the duration of the agonist burst remains constant and only the peak amplitude of the burst is varied (Freund and Büdingen, 1978; Hallett and Marsden, 1979; Hoffman and Strick, 1989, 1990; but see Mustard and Lee, 1987). This pattern of agonist activity has been termed pulse-height modulation (Hoffman and Strick, 1989; see also Gordon and Ghez, 1987). In contrast, when subjects perform elbow or shoulder movements of different amplitudes, both the peak amplitude and the duration of the agonist burst are varied (Wadman et al., 1979; Berardelli et al., 1984; 
Brown and Cooke, 1984; Benecke et al., 1985; Gielen et al., 1985; Cheron and Godaux, 1986; Gottlieb et al., 1989a). Thus, the agonist burst for movements at more proximal joints demonstrates both pulse-height and pulse-width modulation. Furthermore, the rules for controlling agonist muscle activity appear to differ for the skeletomotor and oculomotor systems. Rather than using the pattern of pulsc-hcight modulation scen during many limb movements, oculomotor discharge displays extensive pulse-width modulation during saccadic eye movements of different amplitude (Fuchs and Luschei, 1970; Robinson, 1970; Schiller, 1970; Sindermann et al., 1978).

Gottlieb et al. (1989a) proposed a "dual strategy" hypothesis to explain the diversity of patterns of agonist muscle activity observed in different studies. These authors suggested that when task instructions require explicit control over movement speed, subjects use a "speed-sensitive strategy" in which the central excitatory signals to the motoneuron pools innervating agonist muscles are pulse-height modulated. On the other hand, when task conditions do not require explicit control of speed, subjects use a "speed-insensitive strategy" in which the central excitatory signals to the motoneuron pools are pulse-width modulated. Gottlieb et al. (1989a) were able to place the observations from most prior studies into this framework.

We have proposed an alternative explanation for the apparent differences in the control of distal versus proximal movements (Hoffman and Strick, 1989, 1990). Our explanation is that the pattern of modulation of the agonist burst depends critically upon the force requirements of the task. Specifically, we suggested that when force output cannot be augmented by further pulse-height modulation of the agonist burst, then additional force is generated by pulse-width modulation (see Hoffman and Strick, 1990, p 150; see also Berardelli et al., 1984; Benecke et al., 1985; Cheron and Godaux, 1986; Hoffman and Strick, 1989).

One goal of the present study was to test our hypothesis by applying different loads to step-tracking movements of the wrist. We confirmed our prior observation that the agonist burst in wrist muscles is pulse-height modulated when subjects operate a lightweight manipulandum (Hoffman and Strick, 1990). On the other hand, when subjects operated a heavier manipulandum, the agonist burst displayed an "elbow-like" pattern of activity characterized by both pulse-width and pulse-height modulation. When we applied an even larger load to the wrist, the agonist burst displayed extensive pulse-width modulation, analogous to the modulation of oculomotor discharge seen during saccadic eye movements (Fuchs and Luschei, 1970; Robinson, 1970; Schiller, 1970; Sindermann et al., 1978). Thus, by adjusting the force requirements of the task, we saw that a single wrist muscle could display "wrist-like," "elbow-like," and "eye movement-like" patterns of agonist modulation.

A second goal of the present study was to examine whether pulse-width modulation of the agonist burst could occur even when further increases in force could be accomplished by additional pulse-height modulation of the burst. We examined this possibility by asking subjects to perform wrist movements that required the production of a small, prolonged force. We found that the CNS was able to extend the duration of a small agonist burst, whenever task conditions were appropriate. This result indicates that the CNS can independently control both the amplitude and the duration of the agonist burst.

Preliminary communications of some of this work have appeared previously (Hoffman and Strick, 1989; Hoffman et al., 1990).

\section{Materials and Methods}

Our results arc based on an cxamination of pattcrns of musclc activity in seven normal human subjects (aged $24-41$ years) and in three nonhuman primates (two Macaca mulatta and one Macaca nemestrina). The experiments were conducted according to NIH guidelines and were approved by the institutional committees overseeing human and animal experiments. All of the human subjects gave their informed consent. We will first describe the procedures for the human experiments and then describe the procedures for the monkey studies.

\section{Experiments in human subjects}

Experimental setup and task. Each human subject sat in a chair that supported the forearm and elbow of the dominant (right) limb. The forelimb was gently held in the neutral position (midway between full pronation and full supination). The subject grasped the handle of one of two different manipulanda. The first manipulandum was fully described and illustrated in a prior study (Fig. 1 in Hoffman and Strick, $1986 \mathrm{~b}$ ). The handle of this manipulandum rotated freely about the horizontal and vertical axes. Two potentiometers measured the angles of the wrist in the planes of flexion-extension and radial-ulnar deviation. This manipulandum is a lightweight, low-friction device with a moment of inertia of approximately $0.0025 \mathrm{~kg} \times \mathrm{m}^{2}$ in the radial-ulnar direction. We will refer to this device as the "lightweight manipulandum."

The second manipulandum was coupled to a torque motor (Aeroflex model TQ64W-7HA) and rotated freely only in the vertical plane. A potentiometer was coupled to the rotor of the torque motor to measure the angle of the wrist in the plane of radial-ulnar deviation. The moment of inertia of this manipulandum is approximately $0.005 \mathrm{~kg} \times \mathrm{m}^{2}$. We will refer to this device as the "heavy manipulandum." For some experiments, the torque motor was used to apply elastic loads of $3.0 \mathrm{Nm}$ / $\mathrm{rad}$ or $5.5 \mathrm{Nm} / \mathrm{rad}$ in opposition to radial deviation. In other experiments, we applied a viscoelastic load (viscosity $=0.21 \mathrm{Nm} \mathrm{sec} / \mathrm{rad}$; elasticity $=5.5 \mathrm{Nm} / \mathrm{rad}$ ) in opposition to radial deviation. Thus, three different external load conditions were examined in our experiments: (1) lightweight manipulandum, (2) heavy manipulandum (without additional loads), (3) heavy manipulandum with an additional elastic or viscoelastic load.'

Each subject sat in front of a large screen oscilloscope that displayed a cursor and a target. The cursor moved in proportion to the subject's wrist movements. The target was an open square whose inside diameter equaled $2.5^{\circ}$ of wrist movement. It indicated where the subject should place the cursor. The location of the target on the screen was determined by a DEC PDP $11 / 03$ computer.

Subjects were asked to perform the step-tracking task described in our previous publications (Hoffman and Strick, 1986 b, 1989, 1990). To begin a trial, the subject centered the cursor in the target. The initial target position required $10^{\circ}$ of ulnar deviation of the wrist. After a variable hold period, the target jumped to a new location. The subject, when ready, was required to move the cursor to the new target location by making the appropriate wrist movement. Different target locations required $5^{\circ}, 15^{\circ}$, and $25^{\circ}$ changes in wrist angle in the direction of radial deviation.

Experimental sessions. We gathered data for the human studies in four separate series of experiments that examined three variables: movement amplitude, external load, and movement instruction. Subjects performed the three amplitudes of movement in each experimental series. The first and second series of experiments examined the effects of three different external loads on movements performed "as fast as possible." The third and fourth series of experiments examined the effects of two different prior instructions: "move as fast as possible" and "move as accurately as possible, without overshooting the target." The subjects that participated in each experiment are listed in Table 1. Onc notable featurc of our expcriments is that individual subjects werc studied in a wide range of experimental conditions. As a result, most subjects received considerable practice with the instructions and with the different load conditions. In addition, each series of experiments

\footnotetext{
'These two manipulanda differed not only in their moment of inertia, but also in the extent of wrist fixation required during the performance of the task. When subjects performed radial deviations with the lightweight device, they had to prevent flexion-extension movements. In contrast, the heavy manipulandum rotated only in the radial-ulnar direction. To control for this difference, we examined movement kinematics and muscle activity in two subjects while clamps prevented flexion-extension movements of the lightweight manipulandum. No changes in kinematics or muscle activity were observed.
} 
Table 1. Summary of experiments

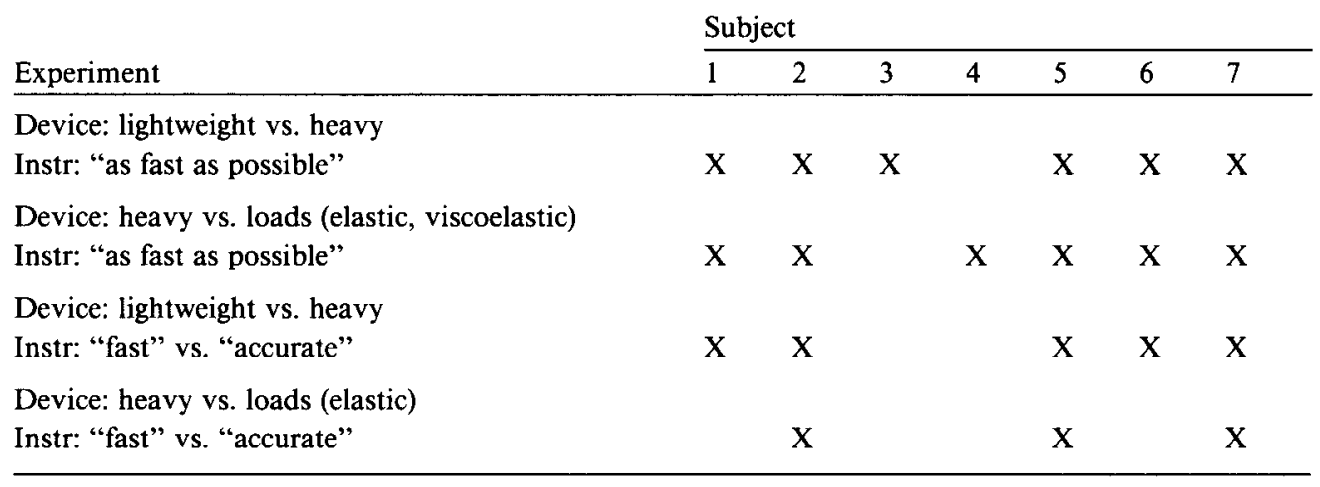

was repeated in some subjects to test the stability of the data. In general, movement kinematics and patterns of muscle activity were quite repeatable.

Movements were performed in blocks of 20 trials in which amplitude, load, and movement instruction were kept constant. After each block, subjects received a $2-4 \mathrm{~min}$ rest period to reduce the possibility of fatigue. When loads were applied by the torque motor, they were presented in the following order: no additional load, smaller elastic load, larger elastic load, no additional load. The second block of trials without additional load was included as a check for fatigue effects. The total number of trials collected in one session varied between 120 and 240 . In each experimental session, subjects performed a block of trials to the $25^{\circ}$ target as fast as possible with the heavy manipulandum. We used this data set as a control to normalize recordings of muscle activity between sessions (see Data analysis).

Data acquisition. Electromyographic (EMG) activity was recorded with surface electrodes (Liberty Mutual Myoelectrodes). The contact surfaces of the electrodes were spaced $1.3 \mathrm{~cm}$ apart. The electrodes were taped on the skin overlying an agonist, extensor carpi radialis longus (ECRL), and an antagonist, extensor carpi ulnaris (ECU). These muscles were selected because their "pulling directions" are very close to radial deviation for ECRL and ulnar deviation for ECU. Furthermore, ECRL has its maximum activity for movements that are close to radial deviation (Hoffman and Strick, 1986a). The electrodes were carefully placed to record large responses with wrist movement and minimal activity with finger movement.

Using surface electrodes spaced $1 \mathrm{~cm}$ apart, we previously observed a single prominent antagonist burst in ECU that began no earlier than the declining phase of the agonist burst (e.g., Figs. 1, 2, 4, and 8 in Hoffman and Strick, 1990). We also have observed single antagonist bursts of comparable latency when recording from other wrist muscles using intramuscular electrodes in humans (e.g., Fig. 2 in Hoffman and Strick, 1986a) and in nonhuman primates (D. S. Hoffman and P. L. Strick, unpublished observations). In the present study, the antagonist burst in ECU was sometimes preceded by muscle activity that began approximately $5-25 \mathrm{msec}$ after the onset of the agonist burst. This early component of activity was more pronounced when movements were opposed by the largest loads. We found that small shifts in electrode position could greatly reduce the amplitude of the early component. Thus, we think that early activity was due to volume conduction from adjacent active muscles (e.g., flexor carpi ulnaris). Consequently, we excluded from analysis all recordings of antagonist activity with an early component larger than $25 \%$ of the peak antagonist burst. It should be noted that an early phase of antagonist activity has been observed in other studies (e.g., Wadman et al., 1979; Mustard and Lee, 1987; Gottlieb et al., 1989b) and has been attributed to the antagonist muscle (see Gottlieb et al., 1989b). However, the presence of this activity in wrist muscles may have been due to the use of surface recordings with interelectrode distances of $3 \mathrm{~cm}$ (Mustard and Lee, 1987).

Amplifiers built into each electrode pair amplified the raw EMG signals by $2666 \times$ or $2800 \times$. These signals were monitored on a storage oscilloscope and were full-wave rectified and filtered $(\tau=10 \mathrm{msec}$; see Gottlieb and Agarwal, 1970). The rectified and filtered signal was digitized at $1.25 \mathrm{kHz}$ by a DEC PDP $11 / 34$ computer. We also digitized position signals from each manipulandum.
Data analysis. The first five trials of each block of 20 were considered practice and were eliminated from further analysis. The remaining 15 trials were examined individually and occasional mistakes (i.e., trials that were slow or inaccurate) were also eliminated. Position and the two EMG signals from the remaining trials were then aligned on movement onset (defined as the first detectable change in the position signal) and averaged. Further analysis was performed on the averaged data.

For each average of movement position, we measured the peak displacement and the duration of the initial trajectory. The duration of the initial trajectory (movement duration) was defined as the time between the first detectable change in position and the earliest peak of displacement. For each average of agonist muscle activity, we measured the peak amplitude and the duration of the agonist burst. The measurement of peak amplitude was limited to the first $65 \mathrm{msec}$ following EMG onset to select only the initial peak of activity. The duration of the burst was measured as the time period when EMG activity was above $25 \%$ of the initial peak amplitude. The $25 \%$ level was selected to eliminate any uncertainty in determining when an agonist burst began or terminated. We measured the peak amplitude and latency of the antagonist burst. The latency was defined as the interval between the onset of the agonist burst and the onset of the antagonist burst. The onsets of the agonist and antagonist bursts were defined as the time when the bursts first reached $25 \%$ of their peak amplitude.

To compare agonist bursts from separate sessions, in each experimental session subjects performed movements to the $25^{\circ}$ target as fast as possible using the heavy manipulandum. We termed the average agonist burst for these trials the "control burst" and set the peak amplitude of this burst equal to $100 \%$. A similar procedure was used for the antagonist burst. However, for this burst, $100 \%$ does not represent a nearly maximal amplitude burst, as it does for the agonist.

We measured the mass of the hand for each subject by determining the volume of water displaced when the hand was immersed up to the center of rotation of the wrist joint. Then, we converted the volume measurement to mass $(1.144 \mathrm{~kg} /$ liter). This resulted in values for hand mass that ranged between 0.275 and $0.484 \mathrm{~kg}$. We also attempted to determine the strength of each subject by asking subjects to produce three maximal radial deviation movements against an external spring $(9.8 \mathrm{~N} / \mathrm{cm})$ attached to the heavy manipulandum. Movement distance was used to calculate maximal torque in the direction of radial deviation. However, this provided an underestimate of maximal torque for the stronger subjects because they were operating at the limit of joint rotation. Even with this underestimate, we found a direct relationship between hand mass and maximal radial torque. The subject with the largest hand (subject 1) developed the largest torque, the subject with the smallest hand (subject 7) developed the smallest torque, and the remaining subjects were intermediate in both hand mass and torque.

\section{Experiments in nonhuman primates}

Each monkey sat in a primate chair with its forearm supported and grasped the handle of a scaled-down version of the lightweight manipulandum. The task that the monkey performed was similar to that in the human study. Monkeys initiated a trial by placing the cursor in the target, which was centered on the screen. The inside diameter of the target measurcd approximately $3.5^{\circ}$ of wrist movement. After a variable 

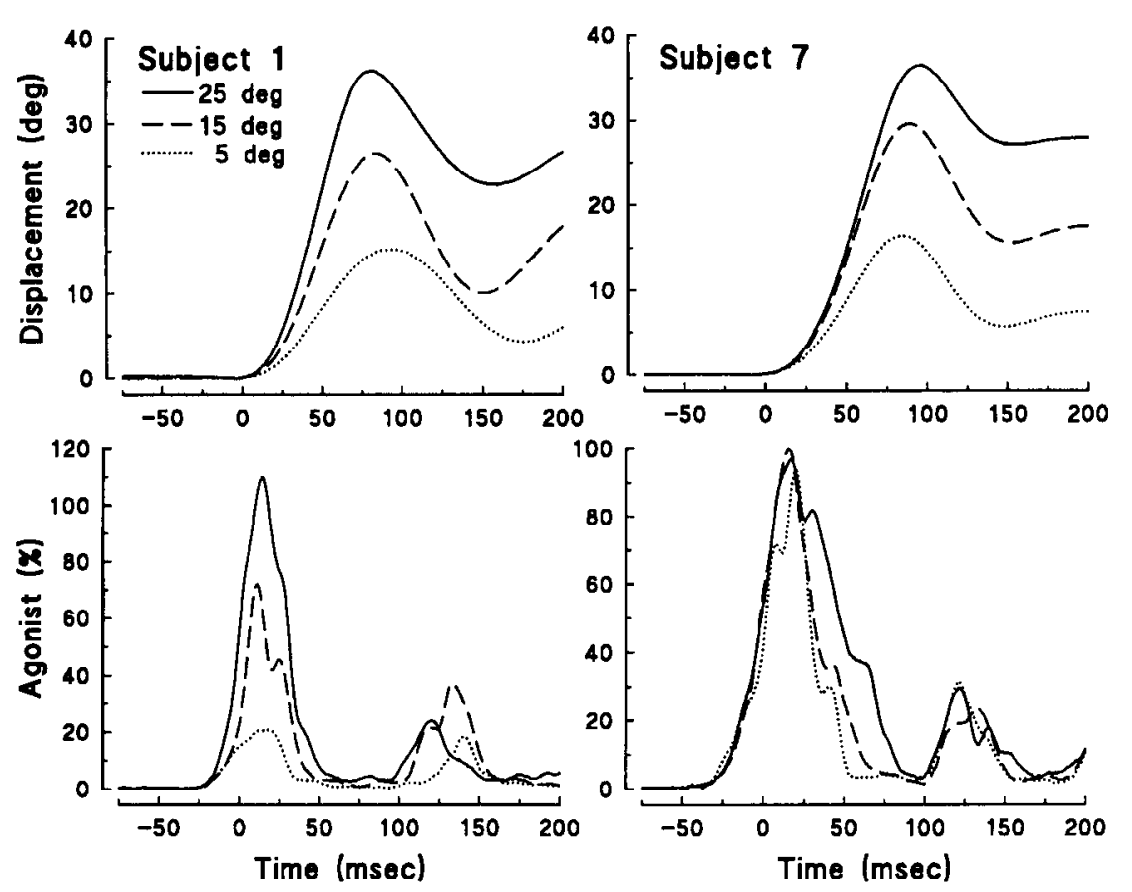

Figure 1. Displacement and agonist muscle activity for wrist movements performed "as fast as possible" with the lightweight manipulandum. Muscle activity was recorded using surface electrodes from ECRL. Each trace is the average of 15 trials. Left, Data from subject 1 , the strongest subject. Right, Data from subject 7 , the weakest subject. The starting position $\left(0^{\circ}\right)$ for the displacement scale represents $10^{\circ}$ of ulnar deviation. The agonist scale was normalized to a control burst, which was defined as the agonist burst for movements to the $25^{\circ}$ target performed "as fast as possible" with the heavy manipulandum.

hold period, the target was stepped from the central position to one of eight different locations equally spaced around the central position. In this study, we will report results only from wrist flexion and extension. Targets required a $20^{\circ}$ change in the angle of the wrist joint. To receive a juice reward, the monkey was required to place the cursor in the new target location with a movement time less than $200 \mathrm{msec}$. Monkeys received considerable training in this task (over 1 year) so that performance was quite stable. One monkey performed movements of different amplitudes $\left(7^{\circ}, 14^{\circ}\right.$, or $21^{\circ}$ changes in wrist angle). In contrast to the human experiments described above, all movements were performed without the addition of any external loads.

EMG recordings were obtaincd from cach monkcy in one or two sessions per week over a 3-4 month time period. During each session, EMG activity was recorded with pairs of single-stranded stainless steel wires (Medwire; 0.003 inch diameter) inserted percutaneously into two different muscles of the forearm. Up to eight different forearm muscles were sampled in each monkey in different sessions. Approximately 1 $\mathrm{mm}$ of the tip of each wire was exposed, and wires were separated by 3-5 mm within each muscle. Each wire was stimulated (10 pulses at $50 / \mathrm{sec}, 100-500 \mu \mathrm{A})$ to confirm that the same movement was evoked following stimulation of each wire and, in most cases, that the same fascicle of the muscle was activated. After amplification, the raw EMG signals were full-wave rectified, filtered, and digitized as in the human experiments described above.

\section{Results}

The effects of changes in load on movements performed "as fast as possible"

\section{Agonist pattern}

"Short-duration" bursts. When most subjects (five of six) operated the lightweight manipulandum, the duration of the agonist burst in wrist muscles was quite brief and did not vary

Table 2. Agonist burst modulation

\begin{tabular}{|c|c|c|c|c|c|c|}
\hline \multirow[b]{2}{*}{ Device } & \multicolumn{2}{|l|}{$5^{\circ}$} & \multicolumn{2}{|l|}{$25^{\circ}$} & \multirow[b]{2}{*}{$\begin{array}{l}\Delta \text { Duration } \\
(\%)\end{array}$} & \multirow[b]{2}{*}{$\underset{(\%)}{n \Delta \text { Amplitude }}$} \\
\hline & $\begin{array}{l}\text { Duration } \\
\text { (msec) }\end{array}$ & $\begin{array}{l}\text { Amplitude } \\
(\%)\end{array}$ & $\begin{array}{l}\text { Duration } \\
\text { (msec) }\end{array}$ & $\begin{array}{l}\text { Amplitude } \\
(\%)\end{array}$ & & \\
\hline \multicolumn{7}{|l|}{ "Fast" movements } \\
\hline Lightweight $(n=5)$ & 51.1 & 47 & 53.9 & 88 & 5 & 87 \\
\hline Subject 7 & 53.7 & 80 & 73.7 & 96 & 37 & 20 \\
\hline Heavy $(n=6)$ & 62.2 & 73 & 86.9 & 100 & 40 & 37 \\
\hline Elastic load $(n=5)$ & 83.4 & 91 & 137.6 & 122 & 65 & 34 \\
\hline $\begin{array}{l}\text { Viscoelastic load } \\
\quad(n=4)\end{array}$ & 77.3 & 87 & 139.3 & 115 & 80 & 32 \\
\hline \multicolumn{7}{|l|}{ "Accurate" movements } \\
\hline Lightweight $(n=4)$ & 49 & 17 & 59.8 & 40 & 22 & 135 \\
\hline Subject 7 & 55.4 & 17 & 98.9 & 23 & 79 & 39 \\
\hline Hcavy $(n=4)$ & 58.7 & 31 & 63.7 & 63 & 9 & 103 \\
\hline Subject 7 & 78.3 & 25 & 112.6 & 77 & 44 & 206 \\
\hline Elastic load $(n=3)$ & 72.1 & 25 & 134.2 & 74 & 86 & 196 \\
\hline
\end{tabular}

Duration = time period when agonist burst was greater than $25 \%$ of its peak amplitude; amplitude: $100 \%=$ amplitude of "control burst" (see Materials and Methods). Elastic load $=5.5 \mathrm{Nm} / \mathrm{rad}$; viscoelastic load $=$ elastic load of $5.5 \mathrm{Nm} /$ $\mathrm{rad}$ and viscous load of $0.21 \mathrm{Nm} \times \mathrm{sec} / \mathrm{rad} ; \Delta$ duration and $\Delta$ amplitude $=\left(25^{\circ}-5^{\circ}\right) / 5^{\circ} \times 100$. 
Figure 2. Displacement and agonist muscle activity for wrist movements performed "as fast as possible" with the heavy manipulandum. Movements were performed by subject 1 , the strongest subject. Left, Heavy manipulandum without additional loads. Right, Heavy manipulandum with an additional elastic load of $5.5 \mathrm{Nm} / \mathrm{rad}$. Muscle activity was recorded from ECRL. Each trace is the average of 15 trials. Scales for displacement and muscle activity are defined in the Figure 1 caption.
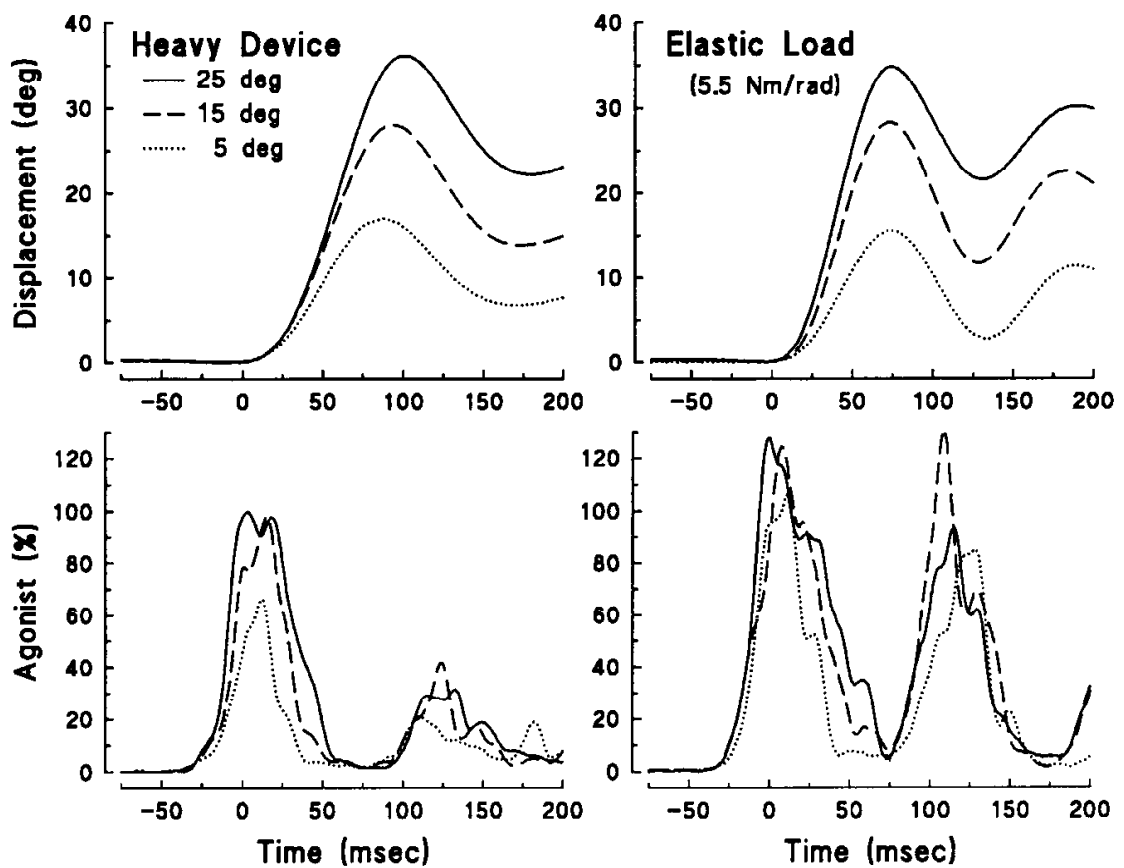

with changes in movement amplitude. The duration of these subjects' bursts averaged $51 \mathrm{msec}$ (range, 46-61 msec) when the target required a $5^{\circ}$ rotation of the wrist and $54 \mathrm{msec}$ (range, $41-71 \mathrm{msec}$ ) when the target required a $25^{\circ}$ wrist rotation (Table 2). "Short-duration" bursts for a single subject varied as little as $2 \mathrm{msec}$ or only as much as $10 \mathrm{msec}$. In contrast, there were marked changes in the peak amplitude of the agonist burst when subjects performed movements to different targets. The best example of a large modulation in the peak amplitude of the agonist burst without a change in burst duration is shown in Figure 1 (left). These observations confirm our prior results using the lightweight manipulandum (Hoffman and Strick, 1990). This device applies only a small load to the wrist (approximately
$0.0025 \mathrm{~kg} \times \mathrm{m}^{2}$ ). Thus, our results suggest that, when the external load is small, subjects vary the force generated by an agonist muscle by modulating only the peak amplitude of a brief agonist burst.

Transition between "short-" and "long-duration" bursts. When we simply asked subjects to perform the same task using the heavy manipulandum, the duration of the agonist burst was noticeably prolonged. For example, the agonist bursts for movements to the $25^{\circ}$ target using the heavy manipulandum were, on average, $33 \mathrm{msec}$ longer than the bursts seen when subjects used the lightweight manipulandum (Table 2). Unlike the results with the lightweight manipulandum, clear modulations in the duration of the agonist burst occurred when subjects performed
Figure 3. Displacement and agonist muscle activity for wrist movements performed "as fast as possible" with the heavy manipulandum. Movements were performed by subject 7 , the weakest subject. Left, Heavy manipulandum without additional loads. Right, Heavy manipulandum with an additional elastic load of $3.0 \mathrm{Nm} / \mathrm{rad}$. Muscle activity was recorded from ECRL. Each trace is the average of 15 trials. Scales for displacement and muscle activity are defined in the Figure 1 caption.
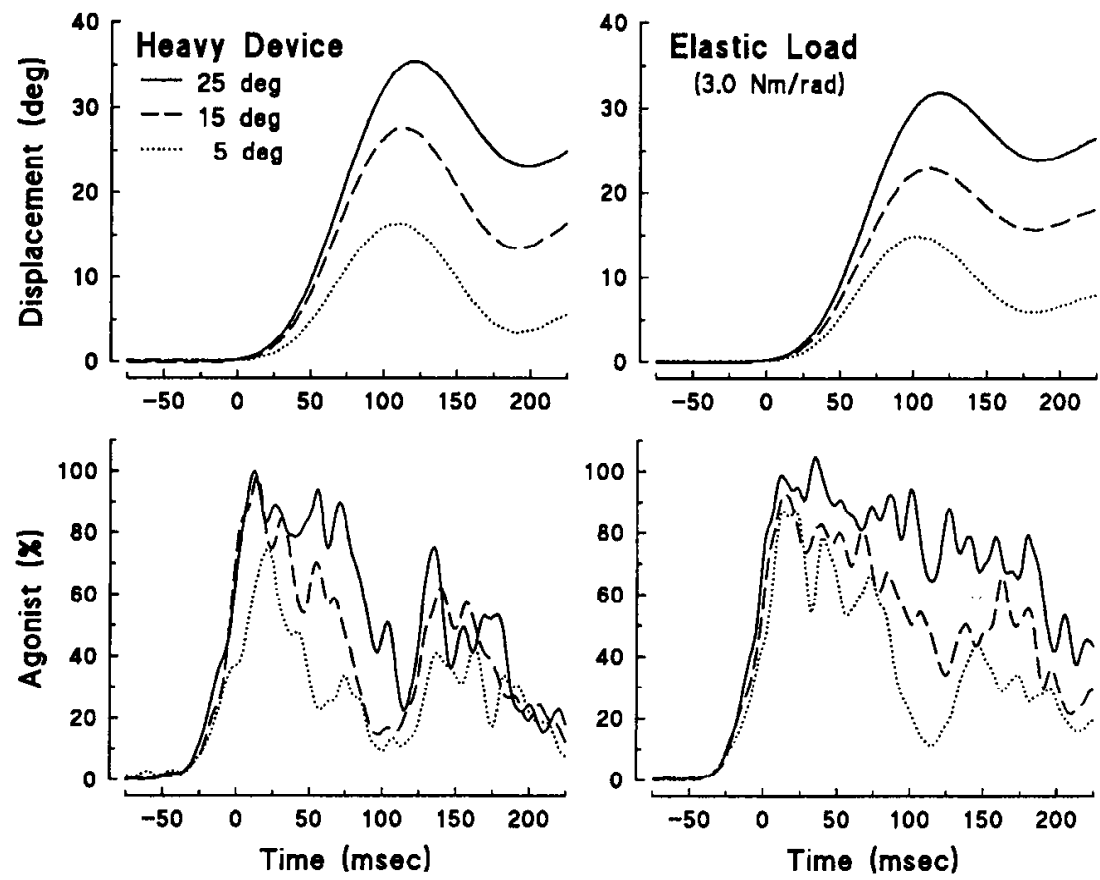
different amplitude movements using the heavy manipulandum (Figs. 2, left; 3, left; see Fig. 6). For example, the duration of the agonist burst averaged $62 \mathrm{msec}$ (range, $43-73 \mathrm{msec}$ ) when the target required a $5^{\circ}$ wrist rotation and $87 \mathrm{msec}$ (range, 54$110 \mathrm{msec}$ ) when the target required a $25^{\circ}$ wrist rotation (Table 2 ). In contrast, modulations in the peak amplitude of the agonist burst were less marked when subjects used the heavy manipulandum than when they used the lightweight manipulandum (Table 2). In fact, the peak amplitude of the agonist burst began to saturate when most subjects performed movements to the $25^{\circ}$ target with the heavy manipulandum (Figs. 2, left; 3, left). Because the heavy manipulandum applied a larger inertial load to wrist movements than the lightweight manipulandum, our results suggest that, at moderate loads, subjects vary the force generated by an agonist muscle by modulating both the peak amplitude and the duration of the agonist burst. Thus, our results demonstrate that the pattern of modulation of the agonist burst can be markedly altered simply by changing the manipulandum that subjects operate, without any change in the instructions to the subject (compare Figs. 1, left, and 2, left; also Figs. 1, right, and 3, left).

"Long-duration" bursts. We observed striking modulations in the duration of the agonist burst when subjects performed different amplitude movements against elastic (and viscoelastic) loads. The duration of the agonist burst averaged $83 \mathrm{msec}$ (range, $50-113 \mathrm{msec}$ ) when the target required a $5^{\circ}$ wrist rotation against the large elastic load and $138 \mathrm{msec}$ (range, 78-211 msec) when the target required a $25^{\circ}$ wrist rotation against the same load (Table 2). We observed agonist bursts with durations as much as 3.9 times longer than the short-duration bursts seen when the same subject operated the lightweight manipulandum (e.g., comparc Figs. 1, right, and 3, right). The large increases in burst duration were associated with only small increments in burst amplitude (e.g., Figs. 2, right; 3, right; see Fig. 8). Thus, the peak amplitude of the agonist burst appeared to approach an asymptote (Fig. 4). These results suggest that when subjects can no longer markedly augment force by increasing the peak amplitude of the agonist burst, further increases in force are produced by extending the duration of the burst.

Two patterns of agonist modulation. A graph of burst duration versus burst amplitude clearly illustrates the two patterns of agonist modulation we observed (Fig. 4). We have placed a vertical dashed line to indicate the upper limit of short-duration bursts (i.e., approximately $1.3 \times$ the minimum duration observed). Agonist bursts to the left of the vertical line displayed largely pulse-height modulation. These bursts had brief, nearly constant durations, and their peak amplitudes were markedly graded. Pulse-height modulation occurred when the task required the agonist muscle to generate relatively low levels of force (e.g., when subjects used the lightweight manipulandum). The agonist bursts to the right of the vertical line displayed pulse-width modulation. The durations of these bursts were prolonged and were markedly graded, and their peak amplitudes were large and only modestly adjusted. Pulse-width modulation occurred when the task required the agonist muscle to generate relatively large amounts of force (e.g., when subjects performed movements against elastic loads). We observed that the transition between these two patterns of modulation was systematic and gradual (Fig. 4).

Intersubject variability. There was considerable variability among subjects in the extent of pulse-height or pulse-width modulation exhibited for a given load condition. This finding

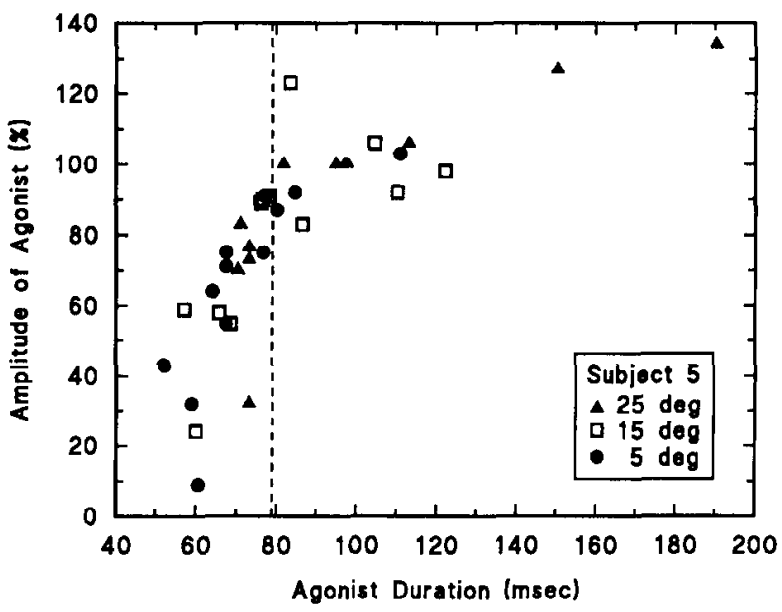

Figure 4. Duration of the agonist burst versus its peak amplitude for a typical subject (subject 5). Each point was measured from averaged agonist bursts for movements performed "as fast as possible" (lightweight manipulandum, heavy manipulandum, and elastic loads) and for "accurate" movements (lightweight manipulandum, heavy manipulandum). Abscissa, Duration of the agonist burst $=$ time period above $25 \%$ of peak amplitude. Ordinate, Amplitude of agonist $=$ peak amplitude of the burst during its initial $65 \mathrm{msec}$. The amplitude of the agonist burst was normalized to a control burst, which is defined in the Figure 1 caption. A vertical dashed line distinguishes "short-duration" bursts on the left from "long-duration" bursts on the right. The line was placed at $1.3 \times$ average minimum duration of the agonist burst for this subject.

is best illustrated by comparing the agonist bursts of the strongest subject (subject 1; see Figs. 1, left; 2) with those of the weakest subject (subject 7; see Figs. 1, right; 3). For most of our load conditions, the strongest subject used pulse-height modulation to grade the force generated by the agonist muscle. The amplitude of the agonist burst began to saturate only when movements were opposed by the larger elastic load. This subject displayed the smallest amount of pulse-width modulation of any subject. The duration of the agonist burst was constant when this subject performed movements of different amplitudes with the lightweight manipulandum (Fig. 1, left), increased slightly (11 msec) for the same task performed with the heavy manipulandum (Fig. 2 , left), and displayed a relatively small change (increasing by $28 \mathrm{msec}$ ) when movements were opposed by the larger elastic load (Fig. 2, right).

In contrast, the weakest subject used pulse-width modulation to grade the force generated by the agonist muscle in all of our load conditions. This subject was the only 1 of 12 , in this or our prior study (Hoffman and Strick, 1990), to modulate the duration of the agonist burst when performing movements of varying amplitude with the lightweight manipulandum (Fig. 1, right; Table 2). The agonist burst displayed extensive pulsewidth modulation when this subject operated the heavy manipulandum (+38 msec; Fig. 3, left) and performed movements against elastic loads (+98 msec; Fig. 3, right). The peak amplitude of the agonist burst appeared to saturate when the weakest subject performed movements with the lightweight manipulandum and displayed little, if any, increase with the addition of greater loads.

There was a striking similarity between the agonist bursts observed in the strongest subject for the largest load condition (Fig. 2, right) and those observed in the weakest subject for the smallest load condition (Fig. 1, right). Furthermore, when we 


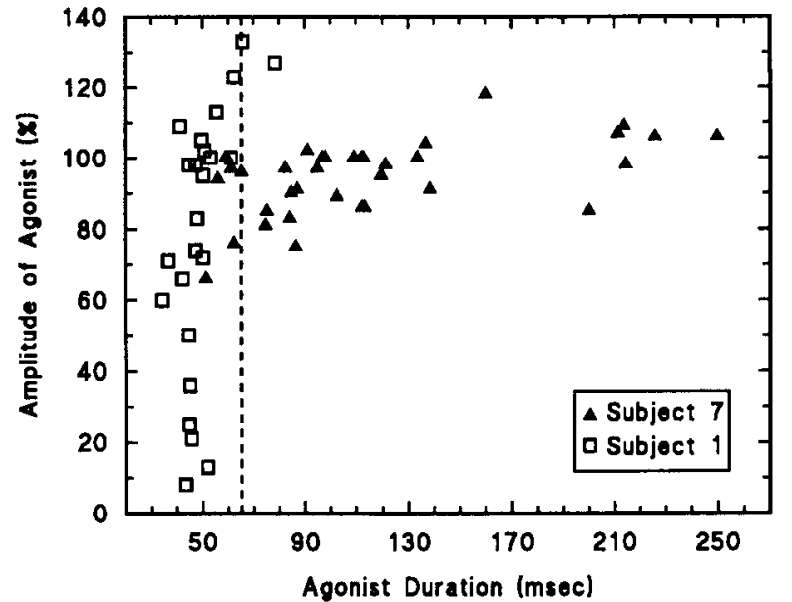

Figure 5. Duration of the agonist burst versus its peak amplitude for subjects 1 and 7 . See Figure 4 for the definitions of the abscissa, ordinate, and the vertical dashed line. The agonist bursts for the "accurate" movements of subject 7 are not included in the graph.
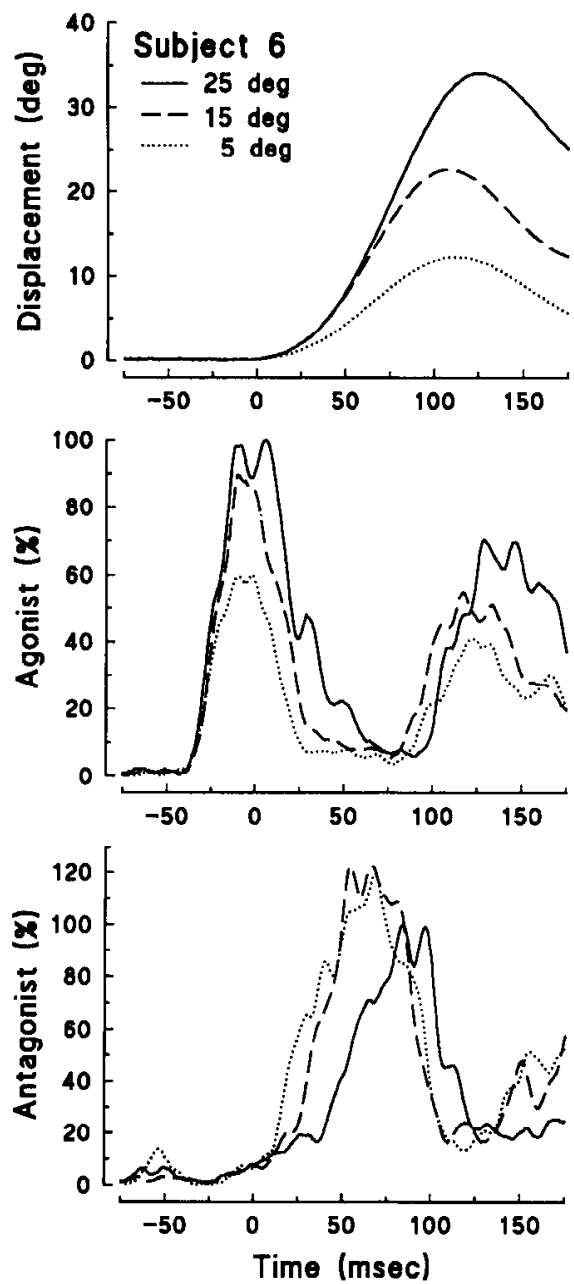

Figure 6. Displacement and muscle activity for wrist movements performed "as fast as possible" with the heavy manipulandum. Movements were performed by subject 6 without any additional loads. Muscle activity was recorded from ECRL (agonist) and ECU (antagonist). Each trace is the average of 15 trials. Scales for displacement and muscle activity are defined in the Figure 1 caption. Antagonist muscle activity also was normalized relative to a control burst. Note that, when the duration of the agonist burst was increased, the antagonist burst was delayed.

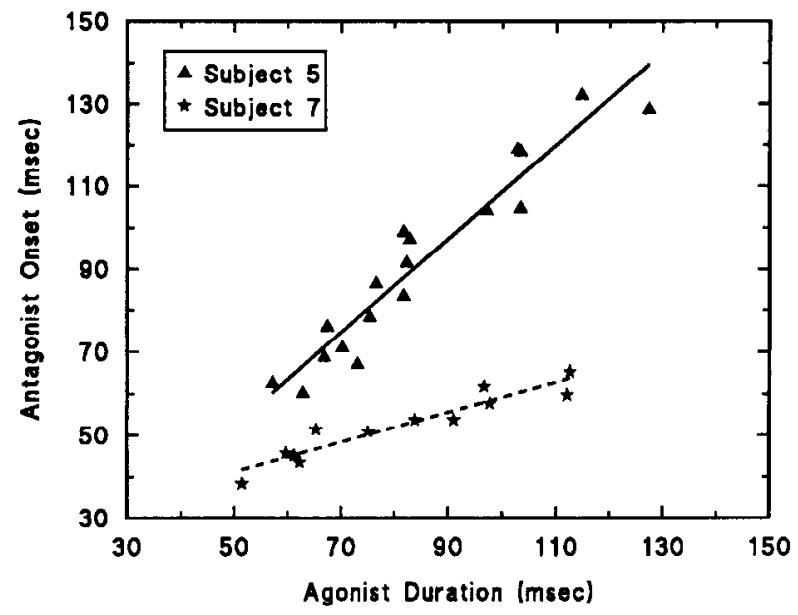

Figure 7. Duration of the agonist burst versus onset of the antagonist burst. The data displayed on this graph were collected while subjects performed movements "as fast as possible" using the lightweight manipulandum, the heavy manipulandum, and the heavy manipulandum with additional clastic loads. Abscissa, Duration of the agonist burst $=$ time period above $25 \%$ of peak amplitude. Ordinate, Onset of the antagonist burst $=$ time interval between $25 \%$ of the maximum agonist burst and $25 \%$ of the maximum antagonist burst. Regression lines: subject $5, y=1.13 x-4.4, r=0.96$; subject $7, y=0.36 x+23.2, r=$ 0.95 .

plotted burst duration versus burst amplitude for these two subjects under the different load conditions (Fig. 5), we found that together, the two sets of points exhibited the full range of agonist modulation seen in the other subjects (compare with Fig. 4). We believe that each subject would have displayed both pulse-height and pulse-width modulation if the load conditions had been appropriate. If this is correct, the strongest subject should demonstrate more extensive pulse-width modulation with further increases in the load opposing wrist movement and the weakest subject should demonstrate pulse-height modulation with decreases in the load. Thus, our observations suggest that differences in the strength of the agonist muscle(s) relative to the experimental load are an important factor leading to intersubject variability in the pattern of agonist modulation.

\section{Antagonist pattern}

We confirmed our prior result that the onset time of the antagonist burst remained constant whenever subjects performed movements with short-duration agonist bursts (see Figs. 2, 4, 8 in Hoffman and Strick, 1990). As notcd abovc, agonist bursts of short-duration were observed in the present study when five of six subjects performed movements with the lightweight manipulandum. On the other hand, when subjects performed movements that resulted in a prolongation of the agonist burst, we found that the antagonist burst was delayed. For example, when all subjects used the heavy manipulandum, the duration of the agonist burst was longer for movements to the $25^{\circ}$ target than for movements to the $5^{\circ}$ target. We found that onset of the antagonist burst was delayed by exactly the same amount as the duration of the agonist burst was increased $(25 \mathrm{msec})$ (e.g., Fig. 6).

We examined the relationship between the onset of the antagonist burst and the duration of the agonist burst for the full range of load conditions in two subjects and found these variables to be highly correlated (Fig. $7 ; r=0.95-0.96$ ). Note that we measured the onset of the antagonist burst relative to the 

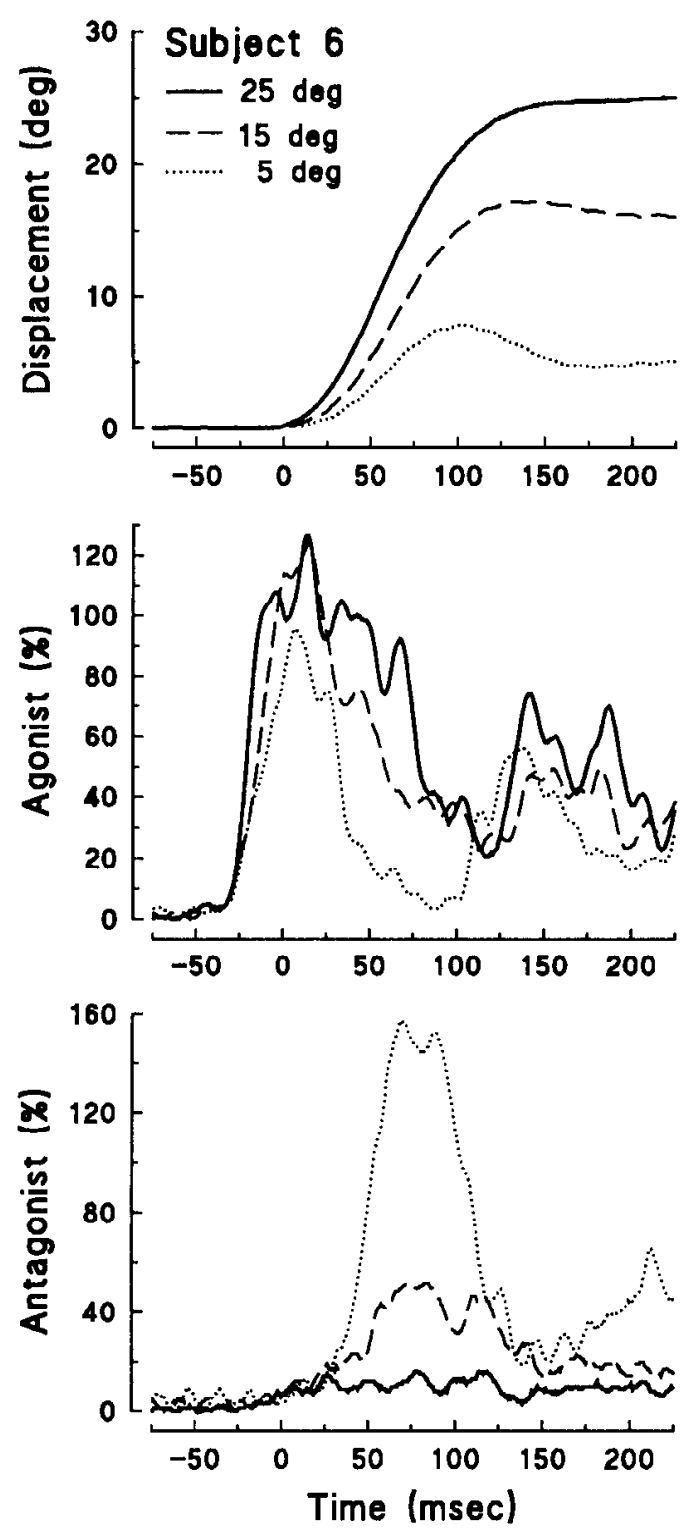

Figure 8. Displacement and muscle activity for wrist movements performed "as fast as possible" against a viscoelastic load by subject 6 . We applied an elastic load of $5.5 \mathrm{Nm} / \mathrm{rad}$ and a viscous load of $0.21 \mathrm{Nm}$ $\mathrm{rad} / \mathrm{sec}$. Muscle activity was recorded from ECRL (agonist) and ECU (antagonist). Each trace is the average of 15 trials. Scales for displacement and muscle activity are defined in the Figure 1 caption. Note the large decrease in the amplitude of the antagonist burst for $15^{\circ}$ movements and the complete absence of the burst for the $25^{\circ}$ movements.

initiation of the agonist burst (see Materials and Methods). The slope of the relation between the duration of the agonist burst and the onset of the antagonist burst was approximately 1 for subject 5 , but was considerably less than 1 for subject 7 . Since the antagonist burst usually was initiated while the agonist burst was declining, a slope less than 1 indicates that, as the agonist burst was lengthencd, coactivation of the agonist and antagonist bursts increased. Overall, our results suggest that the initiation of antagonist activity is linked to a decline in agonist activity.

On the other hand, we did not find a consistent relationship between the peak amplitude of the antagonist burst and any parameter of the agonist burst (either peak amplitude, area, or duration of the burst; e.g., Fig. 6). The peak amplitude of the antagonist burst also was not well related to its time of onset

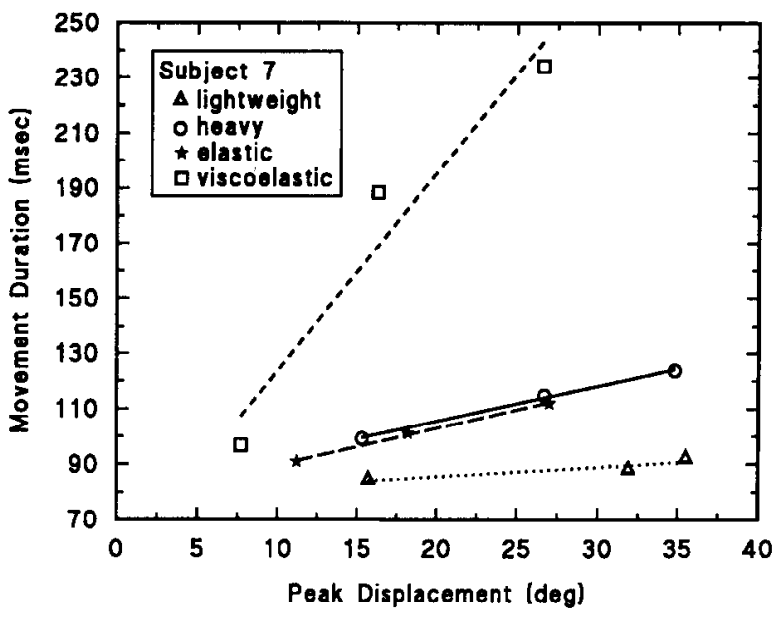

Figure 9. Average peak displacement versus average movement duration for movements performed "as fast as possible" by subject 7 . elastic, elastic load of $5.5 \mathrm{Nm} / \mathrm{rad}$; viscoelastic, elastic load of $5.5 \mathrm{Nm} /$ rad and viscous load of $0.21 \mathrm{Nm} \mathrm{sec/rad.} \mathrm{Regression} \mathrm{lines:} \mathrm{lightweight}$ device, $y-0.34 x+79, r=0.93$; heavy device, $y=1.27 x+80, r=$ 0.998 ; elastic load, $y=1.33 x+76, r=0.999$; viscoelastic load: $y=$ $7.18 x+52, r=0.97$.

(e.g., Fig. 6). However, when the agonist burst was greatly prolonged $(>100 \mathrm{msec}$ ), we observed a consistent reduction or elimination of the antagonist burst (e.g., Fig. 8). These observations indicate that the amplitude of the antagonist burst is determined by processes that differ, in part, from those that specify the magnitude of the agonist burst and also differ from those that specify the timing of the antagonist.

\section{Kinematics}

We confirmed our prior results that movements performed as fast as possible with the lightweight manipulandum exhibited a relatively small change in movement duration with increases in movement amplitude (Fig. 9, Table 3; see also Fig. 5A,B in Hoffman and Strick, 1986b). In contrast, when subjects operated the heavy manipulandum, movement durations were longer than those seen with the lightweight device. In addition, larger movement amplitudes were associated with increases in movement duration that were approximately twice those observed with the lightweight device (Fig. 9, Table 3). Thus, simply changing the device used to monitor movement not only markedly altered the pattern of agonist and antagonist muscle activity, but also resulted in a decreased tendency to keep movement duration constant.

We observed even more dramatic modulations in movement duration when viscoelastic loads were added to the heavy manipulandum (Figs. 8, 9; Table 3). We used this type of load to create mechanical conditions for wrist movements that were more comparable to those encountered for saccadic eye movements. Under this load condition, the duration of wrist movements to the $15^{\circ}$ and $25^{\circ}$ targets was greatly prolonged, especially for the weakest subject (Fig. 9). Larger movement amplitudes were associated with increases in movement duration that averaged almost $10 \times$ those observed with the lightweight device (Fig. 9, Table 3).

\section{EMG patterns in nonhuman primates}

The pattern of activity in wrist and finger muscles of monkeys was quite similar to that in humans performing movements 


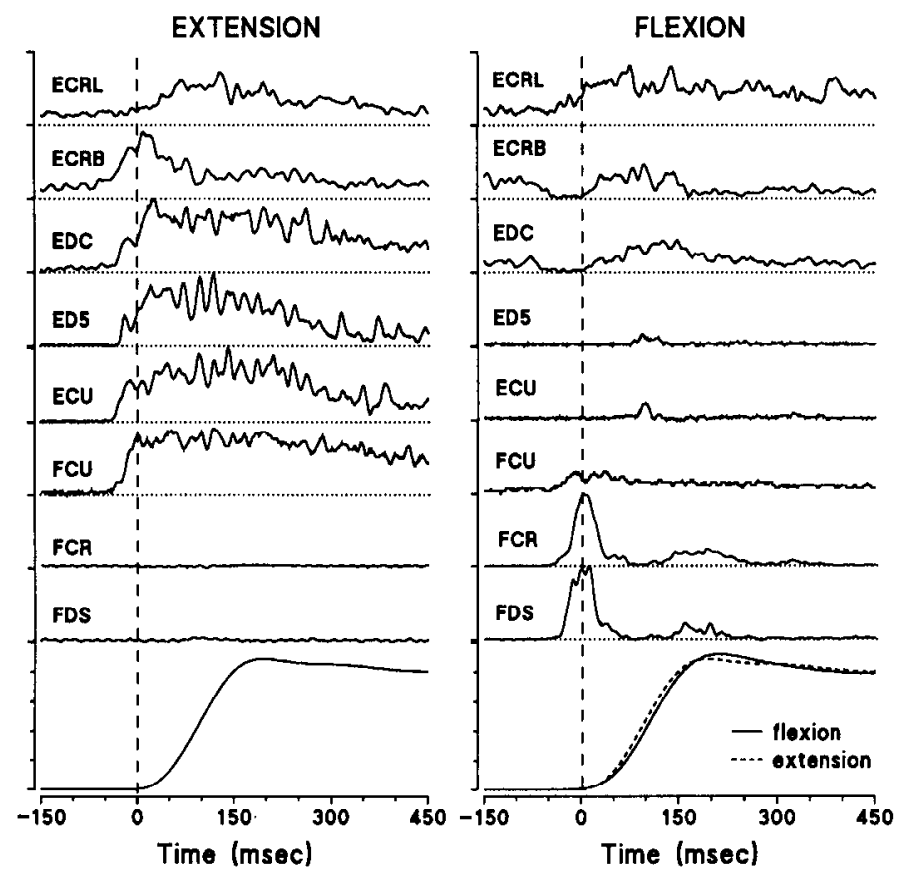

Figure 10. Muscle activity and displacement for wrist extension and flexion in monkey A. Movements were performed from the central position, with a lightweight manipulandum specifically designed for monkeys. The target required a $20^{\circ}$ displacement of the wrist. Left, Muscle activity during wrist extension. Right, Muscle activity during wrist flexion. Note that the patterns of muscle activity are very different for wrist extension versus wrist flexion. Each trace is the average of $7-$ 42 trials (most arc the avcragc of 18-34 trials). For each muscle, 100\% activity $=$ the maximum activity observed from a muscle during the initial $65 \mathrm{msec}$ of activity for any movement direction. Scale for the ordinates is as follows. Extension: ECRL and FCU $=75 \%$; ED5 $=125 \%$; all others $=100 \%$. Flexion: FCR and FDS $=100 \%$; all others $=50 \%$. For displacement, each tick mark, $5^{\circ}$. ECRL, extensor carpi radialis longus; $E C R B$, extensor carpi radialis brevis; $E D C$, extensor digitorum communis; $E D 5$, extensor digiti quinti proprius; $E C U$, extensor carpi ulnaris; $F C U$, flexor carpi ulnaris; $F C R$, flexor carpi radialis; $F D S$, flexor digitorum sublimis.

with different external loads. When monkeys performed steptracking movements using a lightweight manipulandum, wrist flexor muscles displayed "short-duration" agonist bursts during wrist flexion, whereas wrist extensor muscles displayed "longduration" agonist bursts during wrist extension. Short-duration bursts in wrist flexors measured about $55 \mathrm{msec}$ (Fig. 10, right, FCR, FDS; Fig. 11, right). In contrast, during wrist extension each monkey displayed an initial agonist burst in wrist extensors that was quite prolonged (Figs. 10, left; 11, left). This burst measured about $105 \mathrm{msec}$ in monkey C (Fig. 11, left) and several hundred milliseconds in monkeys A and B (e.g., Fig. 10, left, EDC, ED5, ECU).

In monkey $C$, we examined modulation of the peak amplitude and duration of the agonist burst when the animal performed flexion and extension movements of different amplitudes (Fig. 11). For wrist flexion, the duration of the agonist burst in flexor muscles remained relatively constant and only the peak amplitude was altered (Fig. 11, right). This pulse-height modulation of the agonist burst was similar to that observed when most human subjects operated the lightweight manipulandum (e.g., Fig. 1, left). In contrast, both the duration and the peak amplitude of the agonist burst in extensor muscles were altered when the same monkey performed different amplitudes of wrist extension (Fig. 11, left). This combination of pulse-height and pulse-width modulation of the agonist burst was similar to that observed when the weakest human subject operated the lightweight manipulandum (Fig. 1, right) or when other subjects operated the heavy manipulandum (e.g., Figs. 2, left; 6).

It is unlikely that the contrasting patterns of activity in flexor and extensor muscles of the monkey were due to kinematic differences since the movement trajectories for flexion and extension were similar (Fig. 10, right). There is also no reason to believe that the monkeys adopted different "strategies" to perform flexion and extension. Instead, a more likely cause for the activity differences is that the wrist extensor muscles are relatively weaker than the wrist flexor muscles in these nonhuman primates. This explanation is consistent with our conclusion that the strength of the agonist muscle, relative to the external load of the task, determines the extent to which pulse-height or pulse-width modulation of the agonist burst is employed.

\section{Movements performed accurately}

In general, for movements performed as fast as possible, we observed pulse-width modulation of the agonist burst only when its peak amplitude was nearly maximal (i.e., when pulse-height modulation of the burst began to saturate). In the final portion of our study we sought to determine whether pulse-width modulation is limited to this condition. Prior studies have suggested that the accuracy and speed requirements of a task might be

Table 3. Movement kinematics

\begin{tabular}{|c|c|c|c|c|c|}
\hline \multirow[b]{2}{*}{ Device } & \multicolumn{2}{|l|}{$5^{\circ}$} & \multicolumn{2}{|l|}{$25^{\circ}$} & \multirow[b]{2}{*}{$\begin{array}{l}\text { Slope } \\
\text { (msec/degree) }\end{array}$} \\
\hline & $\begin{array}{l}\text { Duration } \\
\text { (msec) }\end{array}$ & $\begin{array}{l}\text { Amplitude } \\
\text { (degrees) }\end{array}$ & $\begin{array}{l}\text { Duration } \\
(\mathrm{msec})\end{array}$ & $\begin{array}{l}\text { Amplitude } \\
\text { (degrees) }\end{array}$ & \\
\hline \multicolumn{6}{|l|}{ "Fast" movements } \\
\hline Lightweight ( $n=5$ ) & 83 & 16.3 & 92 & 34.0 & 0.48 \\
\hline Heavy $(n=5)$ & 96 & 15.2 & 115 & 35.6 & 0.97 \\
\hline Elastic load $(n=4)$ & 88 & 12.6 & 106 & 30.4 & 1.0 \\
\hline Viscoelastic load $(n=3)$ & 91 & 8.8 & 170 & 26.5 & 4.43 \\
\hline \multicolumn{6}{|l|}{ "Accurate" movements } \\
\hline Lightweight $(n=4)$ & 105 & 7.0 & 137 & 26.9 & 1.61 \\
\hline Heavy $(n=3)$ & 113 & 10.3 & 147 & 30.1 & 1.67 \\
\hline Elastic load $(n=3)$ & 115 & 7.6 & 136 & 27.7 & 1.04 \\
\hline
\end{tabular}



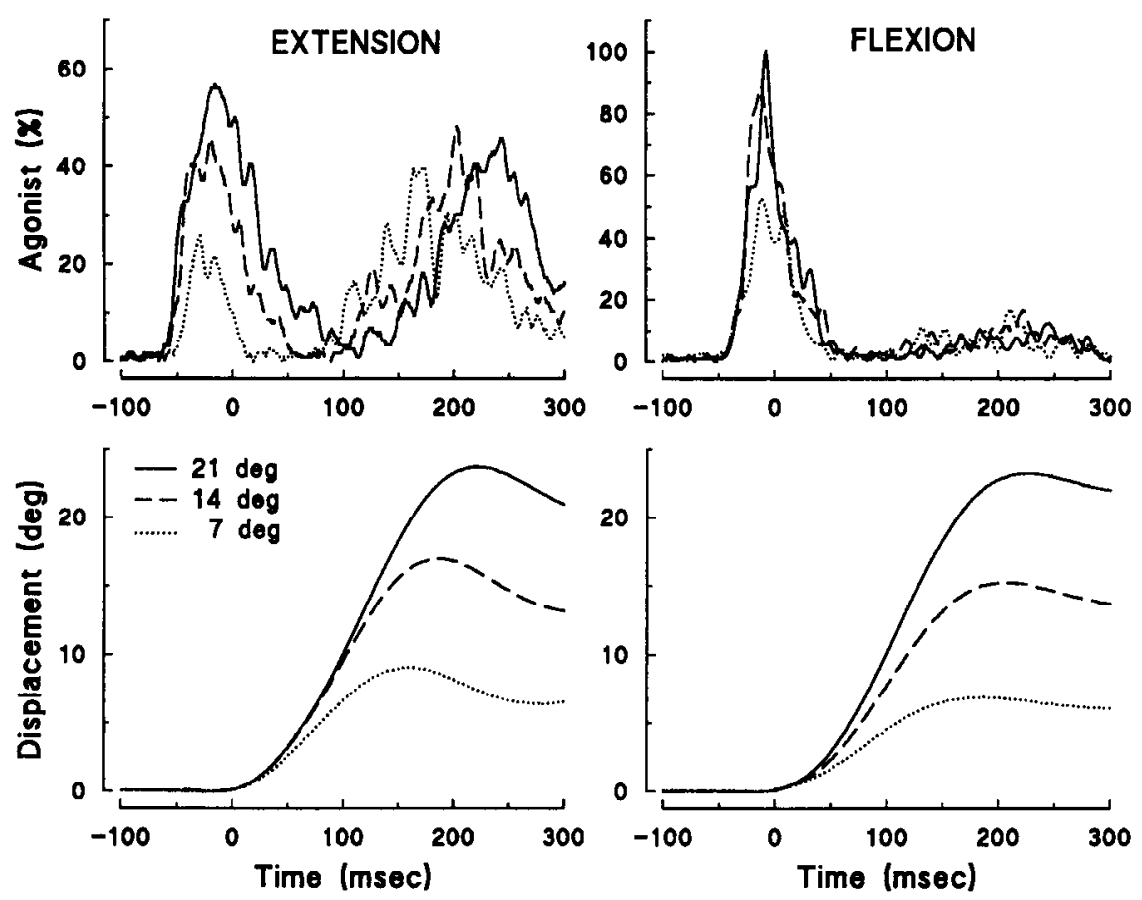

Figure 11. Agonist bursts and displacement for three amplitudes of wrist extension and flexion in monkey $\mathrm{C}$. Targets required $7^{\circ}, 14^{\circ}$, or $21^{\circ}$ displacement of the wrist. Movements were performed using the same manipulandum as in Figure 10. Each trace is the average of 16-23 trials. Left, Agonist bursts in ECU during wrist extension. Right, Agonist bursts in FCR during wrist flexion. Scale for the agonist: $100 \%$ $=$ the maximum activity observed from a muscle during the initial $65 \mathrm{msec}$ of activity.

important factors in determining whether or not pulse-width modulation is observed in agonist muscles (Gottlieb et al., 1989a). Therefore, we instructed subjects to perform step-tracking movements accurately and end their initial trajectory in the target zone without overshoot. With this instruction subjects increased movement duration (Table 3) and generated smaller agonist bursts compared with movements performed "as fast as possible" (e.g., Figs. 12-15, Table 2). ${ }^{2}$

We observed two patterns of modulation of the agonist burst

${ }^{2}$ We did not perform a systematic analysis on the antagonist burst because it was quite small for many of these movements. for "accurate" movements. Under two sets of load conditions (lightweight and heavy manipulandum), most subjects (four of five) demonstrated agonist bursts with short, relatively fixed durations (Figs. 12, 13; Table 2). The length of these shortduration bursts was equal to (Fig. 12) or shorter than (Fig. 13) those observed when subjects moved "as fast as possible." The peak amplitude of these bursts was modulated to vary movement distance (e.g., compare left and right sides of Fig. 12; Table 2). These results confirm our prior observations that the agonist burst can show only pulse-height modulation for changes in intended speed (Hoffman and Strick, 1990).

Under another set of load conditions (elastic loads), all sub-
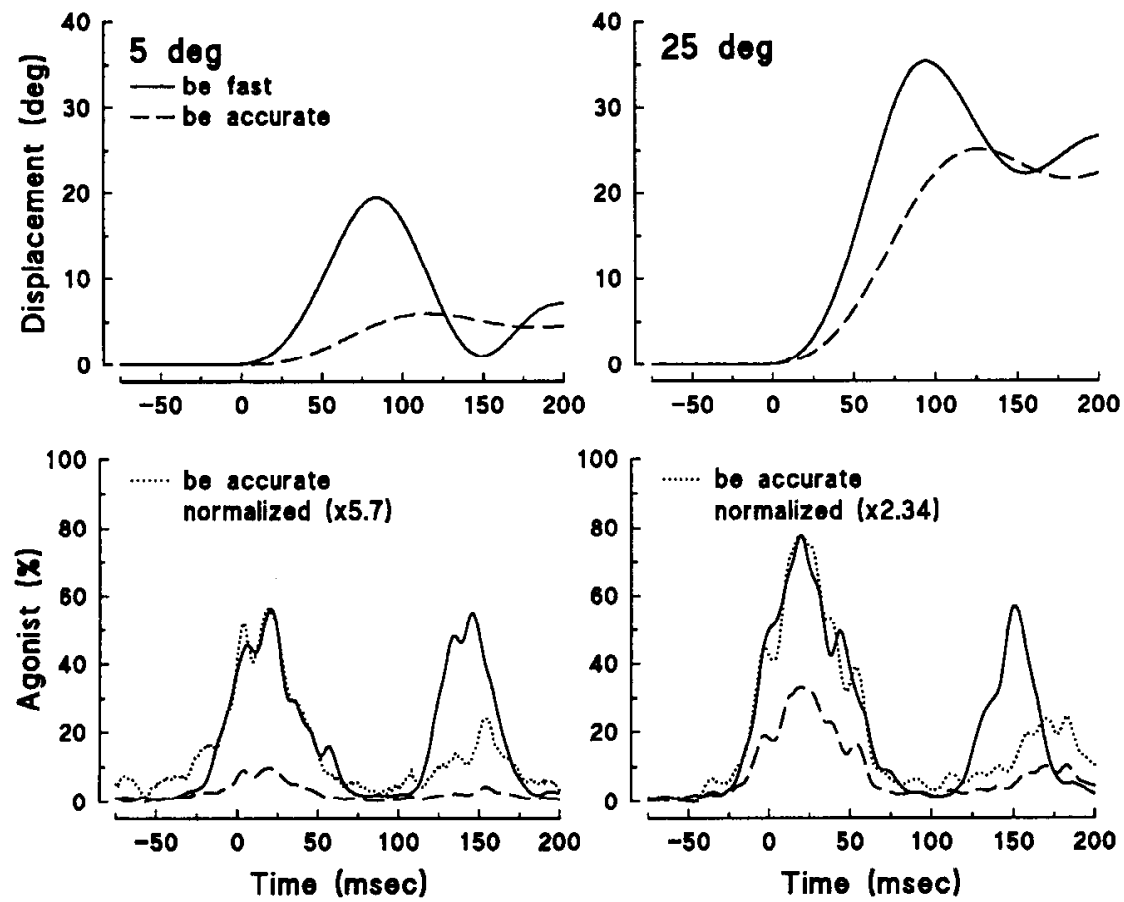

Figure 12. Displacement and agonist muscle activity for wrist movements performed "as fast as possible" or "accurately" with the lightweight manipulandum by a typical subject (subject 5). Each trace is the average of 15 trials. Scales for displacement and muscle activity are defined in the Figure 1 caption. Additionally, the data points for the agonist bursts indicated by the dashed lines were multiplied by a scaling factor to produce the dotted lines. Each scaling factor is given in the figure. Note that the timing of the agonist burst was the same for movements performed "as fast as possible" and "accurately" to a given target. 

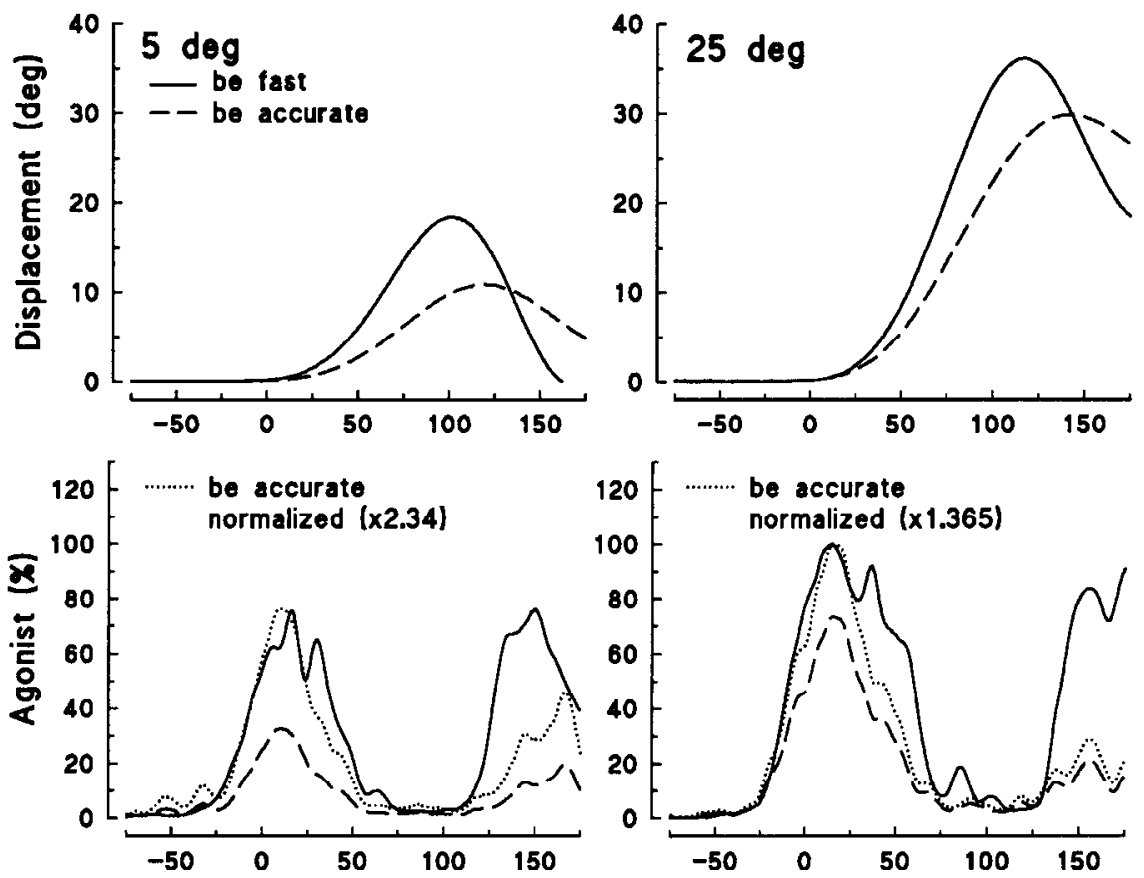

Figure 13. Displacement and muscle activity for wrist movements performed "as fast as possible" or "accurately" with the heavy manipulandum by a typical subject (subject 5 ). See Figure 12 for the meaning of the dotted lines. Note that when the subject performed "accurate" movements, agonist bursts were slightly shorter in duration than when the subject performed movements "as fast as possible." The antagonist burst was absent when the subject performed "accurate" movements to the $25^{\circ}$ target.
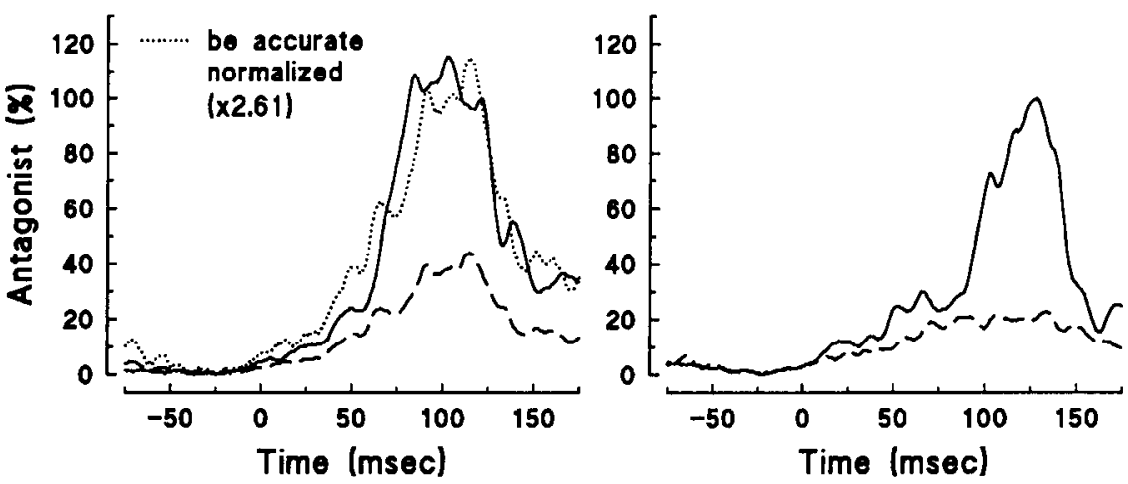

jects performed movements of different amplitudes using both pulse-height and pulse-width modulation (Fig. 14, right, thick dashed line; Fig. 15, stars; Table 2). We observed long-duration agonist bursts even for movements where the peak amplitude of the burst had not reached its maximum. In addition, the weakest subject displayed this pattern of agonist modulation when performing "accurate" movements using the lightweight or heavy manipulandum. Indeed, when this subject performed accurate movements to the $25^{\circ}$ target with the lightweight manipulandum, the peak amplitude of the agonist burst was small (23\% of the control burst) and the duration of the burst was quite long (approximately $2 \times$ the minimum burst duration) (Table 2). This long-duration burst was associated with an unusually long movement duration of $150 \mathrm{msec}$. These findings suggest that the agonist burst is prolonged when there is a need to extend the time course of the propulsive force. This prolongation can occur even though the peak amplitude of the agonist burst is not at its maximum. These observations are significant because they indicate that the nervous system can independently control the duration and the peak amplitude of the agonist burst.

\section{Discussion}

There are two principal findings of the present study. First, we recorded "elbow-like" and "eye movement-like" patterns of activity from wrist muscles when we simply adjusted the load opposing wrist movements. This result suggests that the neural mechanisms for generating distal limb movements may have important similarities to those for generating movements of the proximal limb and eye. Second, we observed that the duration of the agonist burst in wrist muscles could be modified independently from changes in its amplitude. This result suggests that these two variables of agonist activity are separately controlled by the CNS. Our discussion will focus on these two results.

\section{Modulation of the agonist burst: two basic patterns}

When we varied the force requirements of our task, by altering movement amplitude, external load and accuracy instructions, we observed two distinct patterns of modulation of the agonist burst. In one pattern, termed pulse-height modulation, force was graded by varying only the peak amplitude of the burst; the duration of the burst was kept short and nearly constant. We observed this pattern when the force required of the agonist muscle was relatively small, as, for example, when most subjects operated the lightweight manipulandum. Pulse-height modulation also is typical of the agonist muscle activity observed for finger movements (e.g., Hallett and Marsden, 1979).

In the second pattern, termed pulse-width modulation, force was graded by varying the duration of the agonist burst. This pattern of modulation occurred when the peak amplitude of the 


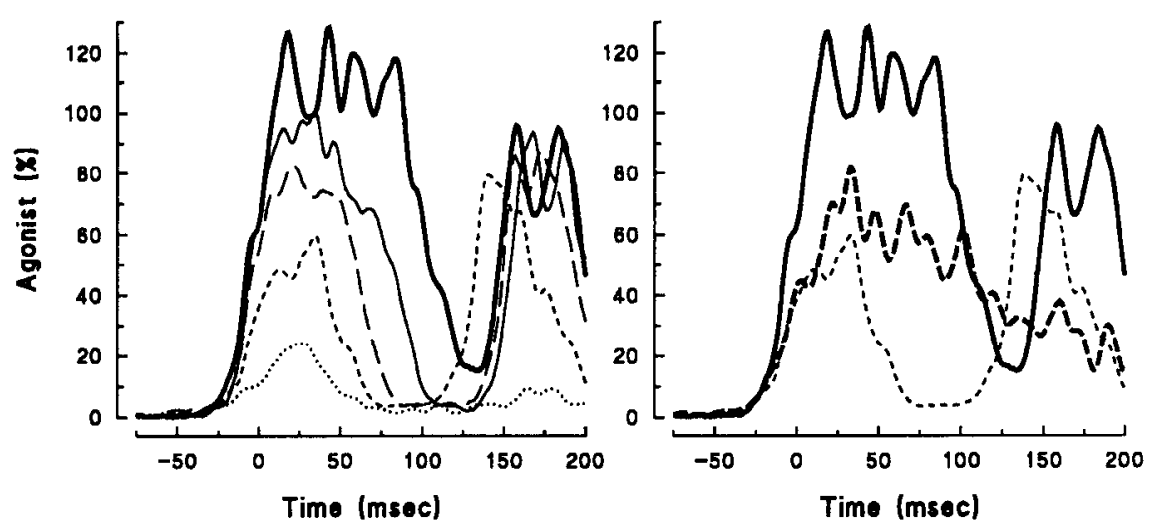

Figure 14. Agonist bursts recorded in a single session when movement amplitude, external load, and movement instructions were varied (subject 5). Left. The progression from pulse-height modulation to the combination of pulse-height and pulse-width modulation of the agonist burst. Bursts that had an amplitude and time course similar to those illustrated are not shown. Right, A clear example of an agonist burst with a prolonged duration, but an amplitude that was not maximal. Each trace is the average of 15 trials. Left: thick solid line, $25^{\circ}$ target, "fast" instruction, elastic load of $3 \mathrm{Nm} / \mathrm{rad}$; thin solid line, $25^{\circ}$ target, "fast" instruction, heavy device; long-dash line, $15^{\circ}$ target, "fast" instruction, heavy device; shortdash line, $5^{\circ}$ target, "fast" instruction, heavy device; dotted line, $5^{\circ}$ target, "accurate" instruction, elastic load of $3 \mathrm{Nm} / \mathrm{rad}$. Right: thick dashed line, $25^{\circ}$ target, "accurate" instruction, elastic load of $3 \mathrm{Nm} / \mathrm{rad}$.

agonist burst was nearly at its maximum. In this circumstance, further increases in force could only be achieved by extensively prolonging the duration of the burst. Thus, we observed pulsewidth modulation when the forces required of the agonist muscle were relatively large, as, for example, when subjects performed movements as fast as possible against elastic or viscoelastic loads. Pulse-width modulation also is typical of the muscle activity observed during shoulder movements (Wadman et al., 1979) and during saccadic eye movements (e.g., Fuchs and Luschei, 1970; Robinson, 1970; Schiller, 1970; Sindermann et al., 1978).

Between these two extreme patterns of agonist modulation, force was graded by varying both the amplitude and the duration of the burst. We observed a combination of pulse-height and pulse-width modulation of the agonist burst when the forces required of the agonist muscle were moderate, as, for example, when most subjects performed movements "as fast as possible" with the heavy manipulandum. The agonist burst observed during elbow movements also is characterized by a combination of pulse-height and pulse-width modulation (e.g., Fig. 6 in Gottlieb et al., 1989a; Fig. 1 in Gottlieb et al., 1989b).

Based on these observations, we conclude that, as the forces required of the agonist muscle increase from low to high, there is a gradual shift from pulse-height to pulse-width modulation of the agonist burst. This conclusion is consistent with prior proposals that the duration of the agonist burst is prolonged when the peak amplitude of the burst can no longer be increased to augment the force output of the agonist muscle (Berardelli et al., 1984; Benecke et al., 1985; Cheron and Godaux, 1986; Fagioli et al., 1988; Hoffman and Strick, 1989). Thus, we propose that many of the diverse patterns of muscle activity observed in studies of step-tracking movements of the limb and eye can be explained by the force requirements of the different tasks uscd in each study, and not by shifts in movement strategy.

\section{Why have results of human studies at the wrist and elbow differed?}

Our results suggest that studies of muscle activity at proximal and distal joints have produced disparate results largely because they have examined agonist muscles in different parts of their operating range. Four main aspects of the experimental con- ditions account for this: movement amplitude, natural inertia of the limb, characteristics of the devices used to monitor movement, and relative muscle strength. To illustrate these experimental factors we will compare our results with the recent findings of Gottlieb and his coworkers (Corcos et al., 1989; Gottlieb et al., 1989a,b, 1990) because the experimental conditions in their studies are particularly well documented.

One experimental condition that influences force requirements of a task is movement amplitude. We observed largely

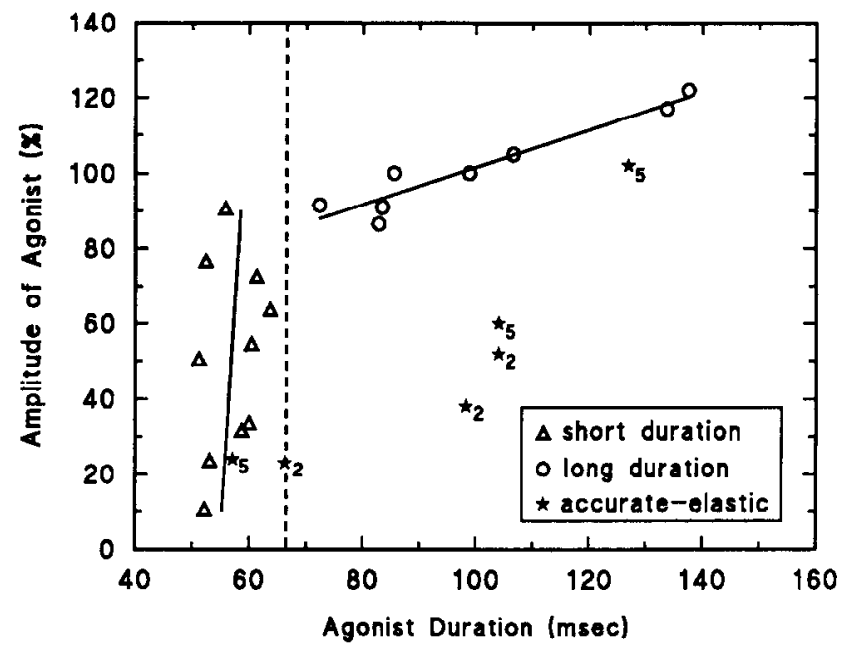

Figure 15. Duration of the agonist burst versus its peak amplitude. For each of the different experimental conditions (movement amplitude external load, and movement instruction), we averaged the duration and the peak amplitude of the agonist bursts across subjects. "Accurate" movements of subject 7 were excluded from the average, because these agonist bursts differed from those of the remaining subjects. Averaged bursts with short durations are indicated by the triangles. Averaged bursts with long durations are indicated by the circles. The stars indicate agonist bursts for "accurate" movements performed against an elastic load of $5.5 \mathrm{Nm} / \mathrm{rad}$ (subject 2) or $3.0 \mathrm{Nm} / \mathrm{rad}$ (subject 5). Abscissa, Duration of the agonist burst $=$ time period abnve $25 \%$ of peak amplitude. Ordinate, Amplitude of agonist $=$ peak amplitude of the burst during its initial $65 \mathrm{msec}$. The amplitude of the agonist burst was normalized to a control burst, which is defined in the Figure 1 caption. A vertical dashed line distinguishes short-duration bursts on the left from long-duration bursts on the right. The line was placed at $1.3 \times$ average minimum duration of the agonist burst. 
pulse-height modulation of the agonist burst for $5-25^{\circ}$ changes in radial deviation of the wrist joint. In contrast, Gottlieb and his colleagues found a combination of pulse-height and pulsewidth modulation for $18-72^{\circ}$ of rotation of the elbow joint. Although it is difficult to compare movement amplitudes at the elbow and the wrist, we estimate that the wrist joint is capable of approximately $80^{\circ}$ of rotation in the radio-ulnar direction and the elbow joint is capable of $160^{\circ}$. Thus, it appears that a similar percentage of the full range of possible joint rotation was examined in both laboratories $(6-31 \%$ at the wrist and $11-$ $45 \%$ at the elbow). In addition, both groups were careful to study movements in the midrange of joint rotation. As a consequence, differences in movement amplitude alone do not appear to account for the contrasting patterns of muscle activity observed for wrist and elbow movements.

Pulse-height modulation of the agonist burst has been observed for elbow movements smaller than $30^{\circ}$ (see Berardelli et al., 1984; Corcos et al., 1992; D. M. Corcos, C.-H. Chen, and G. L. Gottlieb, unpublished observations). In addition, we have observed that the peak amplitude of the agonist burst appeared to saturate and the duration of the burst was prolonged when we examined movements larger than $25^{\circ}$ in some subjects (Hoffman and Strick, unpublished observations). These observations suggest that agonist muscles at the wrist and elbow exhibit pulseheight modulation for "small-amplitude" movements and pulsewidth modulation for "large-amplitude" movements.

A second experimental condition that influences the force requirements of a task is the natural inertia of the limb segment moved during the task. The moment of inertia opposing elbow movements $\left(0.07-0.1 \mathrm{~kg} \times \mathrm{m}^{\prime}\right.$; Gottlieb et al., 1989b) is at least an order of magnitude larger than that opposing wrist movements (about $0.005 \mathrm{~kg} \times \mathrm{m}^{2} ;$ D. S. Hoffman, M. R. Stiles, and P. L. Strick, unpublished observations). A third and closely related experimental condition is the external load opposing or assisting movement. Our lightweight manipulandum increased the moment of inertia opposing wrist movements by about $50 \%$ $\left(0.0025 \mathrm{~kg} \times \mathrm{m}^{2}\right)$, and our heavy manipulandum increased the moment of inertia opposing wrist movements by about $100 \%$ $\left(0.005 \mathrm{~kg} \times \mathrm{m}^{2}\right)$. In contrast, the manipulanda used by Gottlieb and his coworkers increased the moment of inertia opposing elbow movements by approximately $200 \%\left(0.1818 \mathrm{~kg} \times \mathrm{m}^{2}\right.$ in Gottlieb et al., $1989 \mathrm{~b})$ or $100 \%\left(0.0864 \mathrm{~kg} \times \mathrm{m}^{2}\right.$ in Gottlieb et al., 1990). Thus, it is not surprising that the modulation of activity for wrist muscles was "elbow-like" only when most subjects performed movements with the heavy manipulandum. It should be clear from our results that the incrtial charactcristics of the manipulandum significantly influence movement kinematics and patterns of muscle activity.

A fourth factor that influences the pattern of muscle activity observed for a given set of experimental conditions is the relative strength of muscles at the joint examined. In a study of the strength of 12 muscle groups of the arm in normal subjects, Colebatch and Gandevia found that elbow flexors were about $5.5 \times$ stronger than wrist flexors and elbow extensors were about $3.4 \times$ stronger than wrist extensors (Table 1 in Colebatch and Gandevia, 1989). Unfortunately, radial deviation was not specifically examined in this study. Given that the natural inertia opposing elbow movements is more than $10 \times$ greater than that opposing wrist movements, it appears that elbow muscles are "undersized" relative to wrist muscles. This may explain why elbow movements more frequently display pulse-width modulation of the agonist burst than do wrist movements.
A final factor to consider is the duration of the movements that subjects performed. We observed pulse-height modulation of agonist bursts only when movement duration was less than about $150 \mathrm{msec}$. This suggests the possibility that the force resulting from a brief activation of ECRL lasts about $150 \mathrm{msec}$ The duration of the agonist burst will need to be prolonged in order to perform movements with durations longer than the contraction time of the agonist muscle. In the studies of Gottlieb and his colleagues, some movements performed "as fast as possible" had durations of 220-370 msec (Fig. 2 in Gottlieb et al., 1990). By necessity, such movements would require pulse-width modulation of the agonist burst, since the contraction time of elbow flexors is likely to be shorter than these movement durations. When these five factors are considered, our results suggest that appropriate adjustments of the force requirements of the task would lead to comparable patterns of muscle activity for wrist and elbow movements.

\section{Comparison of wrist and eye movements}

There is a longstanding tradition in the study of the central control of movement to consider the systems that control eye and limb movements as different. This legacy is based in part on clear distinctions between the mechanical properties of limb and eye movements. Our results lead us to question the utility of continuing to emphasize the differences between the oculomotor and skeletomotor systems for generating rapid changes in position.

Movements of the eye are opposed by a relatively large inherent viscoelastic resistance and a negligible inertia (Robinson, 1964). As a consequence, saccadic eye movements of greater than about $10^{\circ}$ are generated by a nearly maximal, high-frequency burst of activity in agonist oculomotoneurons, while antagonist motoneurons are nearly silent. Agonist motoneurons display pulse-width modulation; that is, the duration of agonist activity is varied to generate saccadic eye movements of different amplitude (Fuchs and Luschei, 1970; Robinson, 1970; Schiller, 1970). Hence, larger-amplitude saccadic eye movements have a significantly longer duration than smaller-amplitude saccades (2.7 $\mathrm{msec} /$ degree; Baloh et al., 1975), and the peak velocity of large saccades increases only slightly over that of small movements (Boghen et al., 1974; Baloh et al., 1975).

If the mechanical properties of the wrist were like those of the eye, would the patterns of muscle activity and resulting movement kinematics of the two systems be similar? We attempted to address this question by adding elastic and viscoelastic loads to wrist movements. We were, however, limited in our ability to duplicate precisely the mechanical conditions of saccadic eye movements because we could not reduce the inertial load that opposes wrist movements. Nevertheless, when subjects performed larger wrist movements against elastic and viscoelastic loads (e.g., Figs. 3, right; 8), we observed extensive pulse-width modulation of a maximal agonist burst, similar to the modulation of oculomotoneurons described above. In addition, when wrist movements were opposed by viscoelastic loads, the increase in movement duration for larger wrist movements averaged $4.43 \mathrm{msec} /$ degree (Table 3; see also Fig. 9). This is in the same range as that for saccadic eye movements.

It is also important to note that the control of small saccades (less than $10^{\circ}$ ) appears to be quite different from that of large saccades. In fact, small saccadic eye movements share many features in common with step-tracking movements of the wrist. The durations of both oculomotoneuron discharge and the re- 
sulting eye movement are constant for saccades below approximately $10^{\circ}$ (Fuchs et al., 1985). The frequency of oculomotoneuron discharge is modulated to vary the amplitude of small saccades. Furthermore, antagonist eye muscles are weakly active toward the end of small off-saccades (less than $20^{\circ}$ ) in order to assist in braking (Sindermann et al., 1978; Van Gisbergen et al., 1981). These observations suggest that the control of wrist and eye muscles during step-tracking movements may be more similar than previously recognized, since each system displays pulseheight modulation for small forces and pulse-width modulation for larger forces. We do not mean to imply, however, that the control systems for step-tracking movements of the eye and limb are identical. One major difference between the two systems is that subjects are able to vary volitionally the velocity of steptracking movements of the limb to a given target, but lack volitional control over the velocity of a saccade.

\section{Individual differences}

Our study is the first to demonstrate marked differences in patterns of muscle activity between individuals performing a common task (e.g., compare Figs. 2 and 3). The strongest human subject performed most of the movements in our study using only pulse-height modulation of a brief agonist burst. In contrast, the weakest human subject performed the same tasks using pulse-width modulation of a prolonged agonist burst. Despite efforts to change this subject's movement strategy, the pattern of agonist modulation remained unaltered. It is unlikely that the dissimilar patterns of modulation used by the two subjects were due to differences in movement strategy. A simpler explanation is that disparities in muscle strength placed the two individuals in different portions of the operating ranges of their agonist muscles.

Another surprising result of our study was that in nonhuman primates, individual muscles displayed different patterns of modulation when performing similar tasks. We saw that flexor muscles in the monkey demonstrated pulse-height modulation of a brief agonist burst for wrist flexion. This pattern of muscle activity was comparable to that exhibited by our strongest human subject during radial deviation movements. In contrast, extensor muscles in the monkey exhibited pulse-width modulation of a prolonged agonist burst for wrist extension. This pattern of activity was comparable to that exhibited by our weakest human subject during radial deviation movements. It is difficult to conceive that monkeys altered their strategy to perform wrist movements in different directions. Instead, we believe that the different forms of agonist modulation occurred because their extensor muscles are weak compared to their flexor muscles. Therefore, the behavioral task placed these muscles in different portions of their operating range. It is interesting to note that there is also a difference in the strength of corticomotoneuronal and rubromotoneuronal facilitation to wrist flexors and extensors. In general, wrist extensors receive greater amounts of facilitation than flexors (Fetz and Cheney, 1980; Cheney et al., 1991; Mewes and Cheney, 1991). If wrist extensors are weaker than flexors, then perhaps the amount of central excitation to the different motoneuron pools has been adjusted in an attempt to compensate for these differences in strength.

\section{Changes in movement strategy}

We varied movement strategy by asking subjects to perform movements "accurately." Subjects responded to this instruction by decreasing movement velocity, increasing movement dura- tion, and reducing the overshoot of the target. When subjects made movements with either the lightweight or heavy manipulandum, we confirmed our prior observation that subjects use pulse-height modulation of the agonist burst to vary either movement amplitude or intended speed (Hoffman and Strick, 1990). Apparently, the instruction to move accurately caused subjects to slow down and therefore reduced the force requirements of the task. This experiment also demonstrated that the duration of the agonist burst is dissociable from movement duration. Brief agonist bursts were used to generate both fast step-tracking movements with durations of $70-80 \mathrm{msec}$ and slower, more accurate step-tracking movements with durations of 130-140 msec (see also Fig. 8 in Hoffman and Strick, 1990). This result is only possible because the time course of movement is largely determined by adjustments in the amplitude of the antagonist burst (see also Wierzbicka et al., 1986; Ghez and Gordon, 1987; Hoffman and Strick, 1990).

We observed a different result when subjects made "accurate movements" that were opposed by the addition of an elastic load to the heavy manipulandum. All subjects varied the duration of a small agonist burst to adjust movement amplitude. Thus, subjects extended the duration of the agonist burst even when the burst was not at its maximum amplitude. This pattern is similar to the pulse-width modulation of the agonist burst that was reported by Gottlieb et al. (1990) when subjects performed "accurate" elbow movements. In addition, we observed that the weakest subject displayed small, prolonged agonist bursts for "accurate" movements performed with the lightweight manipulandum, when movement duration was $150 \mathrm{msec}$ or longer. Taken logether, these results suggest that subjects can lengthen the duration of a small agonist burst whenever there is a need to extend the time course of the propulsive force. Thus, modulation of the duration of the agonist burst is not limited to those cases when the amplitude of the burst is at or near its maximum. Clearly, our results on accurate movements indicate that the duration of the agonist burst can be controlled independently from its amplitude.

Why is the ability to modulate the duration of submaximal agonist bursts significant? The duration of many rapid stcptracking movements is controlled by modulating an antagonist burst (e.g., Wierzbicka et al., 1986; Ghez and Gordon, 1987; Hoffman and Strick, 1990). This burst appears to be necessary because the propulsive force produced by an agonist burst of minimum duration far outlasts the time course of the fastest wrist movements (58-71 msec) (see discussion in Hoffman and Strick, 1990). As movement duration is prolonged, there is less need for the braking force generated by the antagonist (Lestienne, 1979). At the point where the duration of a step-tracking movement exceeds the contraction time of the agonist muscle, further increases in movement duration can only be accomplished by prolonging the duration of the agonist burst. Thus, the ability to modulate the duration of a small-amplitude agonist burst provides the nervous system with the flexibility to generate step-tracking movements with a wide range of intended speeds.

Taken together, our observations have many similarities to those of Gottlieb and his coworkers. For example, both groups have observed that there are two ways of modulating the agonist burst (intensity and duration) and that pulse-width modulation of the agonist burst can occur when burst amplitude is either large or small. However, the terms "speed sensitive" and "speed insensitive" lead to considerable confusion when generally applied to the control of movement. For example, subjects showed 
pulse-height modulation of the agonist burst (part of the speedsensitive strategy) for rapid wrist movements of different amplitude. According to Gottlieb et al. (1989a), this task should have required the use of the speed-insensitive strategy (i.e., pulse width modulation of the agonist burst). Furthermore, when the same subjects performed rapid wrist movements of different amplitude that were opposed by an elastic load, the agonist burst showed pulse-width modulation. We see no utility in conceiving of this alteration of muscle activity as a switch in strategy. As noted above, we also see no reason to consider the differences between monkey wrist flexors and extensors as reflecting the use of dissimilar strategies. Thus, we believe that the terms "speed insensitive" and "speed sensitive" are not generally applicable and that modulations in agonist and antagonist activity should be interpreted in terms of the force requirements of the task.

\section{Relations between agonist and antagonist muscle activity}

In a prior study we demonstrated that the amplitude of the antagonist burst could be modulated independently of the amplitude of the agonist burst (see Fig. 8 in Hoffman and Strick, 1990; see also Ghez and Gordon, 1987). This result indicated that the mechanism that generates the antagonist burst is, in part, separate from that which produces the agonist burst (see Hoffman and Strick, 1990, p 151). In the present study, we found that the duration of the agonist burst and the onset of the antagonist burst were strongly correlated. This result supports prior proposals of a coupling between the initiation of the antagonist burst and the termination of the agonist burst (see also Lestienne, 1979; Cheron and Godaux, 1986; Ghez and Gordon, 1987). Clearly, the amplitude and timing of the agonist and antagonist bursts must be carefully coordinated in order to apply the appropriate amount of "central braking" necessary to terminate a rapid step-tracking movement.

\section{Concluding remarks}

The results of this study, together with our prior study (Hoffman and Strick, 1990), indicate that the central control over agonist and antagonist muscle activity is impressive in its flexibility. The nervous system can independently specify three parameters of agonist and antagonist muscle activity: (1) the amplitude of an agonist burst, (2) the duration of an agonist burst, and (3) the amplitude of an antagonist burst. This flexibility over the control of agonist and antagonist activity enables the nervous system to shape precisely the magnitude and time course of the force needed to accomplish a specific task.

Future studies will need to investigate the origin of the descending command signals for step-tracking movements and how these signals combine with spinal mechanisms to generate agonist and antagonist bursts. As a start, one could ask how the nervous system produces the different patterns of agonist activity in wrist flexors and extensors in nonhuman primates. Another important issue is whether the central neurons that gencrate the agonist burst in a muscle also are responsible for producing the antagonist burst in the same muscle. The resolution of these important issues will require neuron recording studies in awake trained primates.

\section{References}

Baloh RW, Sills AW, Kumley WE, Honrubia V (1975) Quantitative measurement of saccade amplitude, duration, and velocity. Neurology 25:1065-1070.

Benecke R, Meinck H-M, Conrad B (1985) Rapid goal-directed elbow flexion movements: limitations of the speed control system due to neural constraints. Exp Brain Res 59:470-477.

Berardelli A, Rothwell JC, Day BL, Kachi T, Marsden CD (1984) Duration of the first agonist EMG burst in ballistic arm movements. Brain Res 304:183-187.

Boghen D, Troost BT, Daroff RB, Dell'Osso LF, Birkett JE (1974) Velocity characteristics of normal human saccades. Invest Ophthalmol 13:619-623.

Brown SH, Cooke JD (1984) Initial agonist burst duration depends on movement amplitude. Exp Brain Res 55:523-527.

Cheney PD, Mewes K, Widener G (1991) Effects on wrist and digit muscle activity from microstimuli applied at the sites of rubromotoneuronal cells in primates. J Neurophysiol 66:1978-1992.

Cheron G, Godaux E (1986) Sclf-terminated fast movement of the forearm in man: amplitude dependence of the triple burst pattern. $J$ Biophys Biomec 10:109-117.

Colebatch JG, Gandevia SC (1989) The distribution of muscular weakness in upper motor neuron lesions affecting the arm. Brain 112 : 749-763.

Corcos DM, Gottlieb GL, Agarwal GC (1989) Organizing principles for single joint movements. II. A speed-sensitive strategy. J Neurophysiol 62:358-368.

Corcos DM, Gottlieb GL, Chen C-H (1992) Modifications in the speed-insensitive strategy for movements requiring low levels of force. Soc Neurosci Abstr 18:1053.

Fagioli S, Berardelli A, Hallett M, Accornero N, Manfredi M (1988) The first agonist and antagonist burst in patients with an upper motor neuron syndrome. Movement Disord 3:126-132.

Fetz EE, Cheney PD (1980) Postspike facilitation of forelimb muscle activity by primate corticomotoneuronal cells. J Neurophysiol 44: 751-772.

Freund H-J, Büdingen HJ (1978) The relationship between speed and amplitude of the fastest voluntary contractions of human arm muscles. Exp Brain Res 31:1-12.

Fuchs AF, Luschei ES (1970) Firing patterns of abducens neurons of alert monkeys in relationship to horizontal eye movement. J Neurophysiol 33:382-392.

Fuchs AF, Kaneko CRS, Scudder CA (1985) Brainstem control of saccadic eye movements. Annu Rev Neurosci 8:307-337.

Ghez C, Gordon J (1987) Trajectory control in targeted force impulses. I. Role of opposing muscles. Exp Brain Res 67:225-240.

Giclen CCAM, van den Oosten K, Pull ter Gunne F (1985) Relation between EMG activation patterns and kinematic properties of aimed arm movements. J Mot Behav 17:421-442.

Gordon J, Ghez C (1987) Trajectory control in targeted force impulses. II. Pulse height control. Exp Brain Res 67:241-252.

Gottlieb GL, Agarwal GC (1970) Filtering of electromyographic signals. Am J Phys Med 49:142-146.

Gottlieb GL, Corcos DM, Agarwal GC (1989a) Strategies for the control of voluntary movements with one mechanical degree of freedom. Behav Brain Sci 12:189-250.

Gottlieb GL, Corcos DM, Agarwal GC (1989b) Organizing principles for single joint movements. I. A speed-insensitive strategy. J Neurophysiol 62:342-357.

Gottlieb GL, Corcos DM, Agarwal GC, Latash ML (1990) Organizing principles for single joint movements. III. The specd-insensitive strategy as default. J Neurophysiol 63:625-636.

Hallett M, Marsden CD (1979) Ballistic flexion movements of the human thumb. J Physiol (Lond) 294:33-50.

Hoffman DS, Strick PL (1986a) Activity of wrist muscles during steptracking movements in different directions. Brain Res 367:287-291.

Hoffman DS, Strick PL (1986b) Step-tracking movements of the wrist in humans. I. Kinematic analysis. J Neurosci 6:3309-3318.

Hoffman DS, Strick PL (1989) Force requirements and patterns of muscle activity. Behav Brain Sci 12:221-224.

Hoffman DS, Strick PL (1990) Step-tracking movements of the wrist in humans. II. EMG analysis. J Neurosci 10:142-152.

Hoffman DS, Stiles MR, Strick PL (1990) Force requirements determine the pattern of agonist modulation. Soc Neurosci Abstr 16:1089.

Lestienne F (1979) Effects of inertial load and velocity on the braking process of voluntary limb movements. Exp Brain Res 35:407-418.

Mewes K, Cheney PD (1991) Facilitation and suppression of digit and wrist muscles from single rubromotoneuronal cells in the awake monkey. J Neurophysiol 66:1965-1977.

Mustard BE, Lee RG (1987) Relationship between EMG patterns and 
kinematic properties for flexion movements at the human wrist. Exp Brain Res 66:247-256.

Robinson DA (1964) The mechanics of human saccadic eye movement. J Physiol (Lond) 174:245-264.

Robinson DA (1970) Oculomotor unit behavior in the monkey. J Neurophysiol 33:393-404.

Schiller PH (1970) The discharge characteristics of single units in the oculomotor and abducens nuclei of the unanesthetized monkey. Exp Brain Res 10:347-362.

Sindermann F, Geiselmann B, Fischler M (1978) Single motor unit activity in extraocular muscles in man during fixation and saccades. Electroencephalogr Clin Neurophysiol 45:64-73.
Van Gisbergen JAM, Robinson DA, Gielen S (1981) A quantitative analysis of generation of saccadic eye movements by burst neurons. J Neurophysiol 45:417-442.

Wadman WJ, Denier van der Gon JJ, Geuze RH, Mol CR (1979) Control of fast goal-directed arm movements. J Hum Mov Stud 5:317.

Waters P, Strick PL (1981) Influence of "strategy" on muscle activity during ballistic movements. Brain Res 207:189-194.

Wierzbicka MM, Wiegner AW, Shahani BT (1986) Role of agonist and antagonist muscles in fast arm movements in man. Exp Brain Res 63:331-340. 